\title{
The Role of Oxygen Intake and Liver Enzyme on The Dynamics of Damaged Hepatocytes: Implications to Ischaemic Liver Injury via A Mathematical Model
}

\author{
Aditi Ghosh ${ }^{1, *}$ Leon Arriola ${ }^{1}$, Andrew Mason ${ }^{1}$, \\ William Lee ${ }^{2}$, Anuj Mubayi ${ }^{3}$ \\ ${ }^{1}$ Department of Mathematics, University of Wisconsin - Whitewater, Whitewater, WI 53190, USA: \\ A.G. email: ghosha@uww.edu \\ ${ }^{2}$ Department of Hepatology, University of Texas Southwestern Medical Center TX 75390, USA \\ ${ }^{3}$ Simon A. Levin Mathematical, Computational and Modeling Sciences Center, \\ School of Human Evolution and Social Change, Arizona State University, Tempe, AZ 85287, USA
}

March 9, 2020

\begin{abstract}
Ischaemic Hepatitis ( $\mathrm{IH})$ or Hypoxic Hepatitis (HH) also known as centrilobular liver cell necrosis is an acute liver injury characterized by a rapid increase in serum aminotransferase. The liver injury typically results from another underlying medical conditions like cardiac failure, respiratory failure and septic shock in which the liver becomes damaged due to deprivation of either blood or oxygen. IH is a potentially lethal condition which is often preventable if diagnosed properly. Unfortunately, mechanism that causes IH are often not well understood, making it difficult to diagnose or accurately quantify the patterns of related biomakers. In most cases, currently the only way to determine a case of IH (i.e., to diagnose it) is to rule out all other possible conditions for other liver injuries. A better understanding of the liver's response to IH is necessary to aid in its diagnosis, measurement and improve outcomes. The goal of this study, is to identify mechanisms that can alter a few associated biomarkers for reducing density of damaged hepatocytes, and thus reduce chances of $\mathbf{I H}$. To this end, we develop a mathematical model capturing dynamics of hepatocytes in the liver through the rise and fall of associated liver enzymes aspartate transaminase (AST), alanine transaminase (ALT) and lactate dehydrogenase (LDH) related to condition of IH. The model analysis provides a novel approach to predict the level of biomarkers given variations in the systemic oxygen in the body. Using IH patient data in US, novel model parameters are described and then estimated for the first time to capture real time dynamics of hepatocytes in the presence and absence of IH condition. Different scenarios of patient conditions were also analyzed and validated using empirical information. This study and its results may allow physicians to estimate the extent of liver damage in a $\mathrm{IH}$ patient based on their enzyme levels and receive faster treatment on real time basis.
\end{abstract}

\section{Introduction}

Ischaemic Hepatitis (IH) is a critical liver injury due to centrilobular liver cell necrosis with a massive increase in serum aminotransferase $[18,20]$. It accounts for the most frequent cause of liver injury with $10 \%$ of patients admitted to hospital intensive care units $[13,37,40]$. Cell necrosis in Ischaemic Hepatitis is generally due to low oxygen extraction by hepatocytes, hepatic blood perfusion, systemic arterial hypoxemia and venous congestion [13]. The cause of IH is due to an underlying condition such as shock, trauma, or surgery. This underlying condition could be sudden, as is the case with shock

\footnotetext{
${ }^{*}$ Corresponding author; , Claire Onsager ${ }^{1}$,
} 
(cardiac, allergic, hypovolemic, or septic) or could be a chronic condition such as heart failure [17]. Heart failure damages the blood flow through the portal vein, which in turn increases the hepatic vein's blood flow. The damage to the veins occurs over time without causing any immediate injury to the liver. For example, heart failure can result in a condition called congestive hepatopathy. Over a long period of time, this increased blood flow to the hepatic vein causes congestion of the vein, eventually building to a point of failure resulting in IH $[25,30,44]$. For this reason, there are no chronic cases of $\mathrm{IH}$, just chronic conditions that can cause it. In addition to the causes, there are also other various conditions that can increase the risk for IH because they weaken the blood flow systems to the liver. A few such conditions are kidney disease, heart disease, and liver disease [11,17, 42].

The liver is supplied with two types of blood delivery to protect against oxygen impairment through two different veins namely the portal vein and the hepatic vein. The portal vein delivers two thirds of the liver's blood. Despite this, the portal vein only provides $50 \%$ of the oxygen needed. The other $50 \%$ is provided by the oxygen rich hepatic blood. In order to protect itself against damage, the liver has a process called the 'hepatic arterial buffer response' $[18,19]$. Through this process, damage to the portal vein results in an increase in blood flow through the hepatic vein. Similarly, damage to the hepatic vein leads to an increase in the oxygen levels of the blood through the portal vein. This extra layer of protection implies that significant injury to one or both the portal vein system and arterial hepatic system is necessary to cause ischaemic hepatitis. The damage must be severe enough that it reduces the percentage of oxygen received in the liver $[1,8,43]$. Oxygen delivery (DO2) is the volume of oxygen delivered to the systemic vascular bed per minute. Systemic oxygen delivery (DO2) in the body depends on several factors like cardiac output, haemoglobin level and oxygen saturation of haemoglobin. Normal value for $\left(D_{2}\right)$ lies within 520 and $720 \mathrm{mlO} / \mathrm{min} \cdot \mathrm{m}^{2}$ and often low cardiac output can bring down $\left(\mathrm{DO}_{2}\right)$ to the critical threshold for hypoxia which is 330 $\mathrm{mlO} O_{2} / \min . \mathrm{m}^{2}[18,19,21,24,26,31]$. This work here focuses on developing a mathematical model to understand liver damage due to IH with respect to systemic oxygen delivery (DO2) in the body.

Once IH occurs, it is characterized by abnormal liver function, tissue damage, and tissue death that leads to liver failure. There is also a rapid rise in various serum levels such as aspartate transaminase (AST), alanine transaminase (ALT) and lactate dehydrogenase (LDH) $[18,22,34,45]$. These elevated levels are often the first sign of liver injury. Once this is realized, physicians determine the type of liver injury that has occurred. Typically there is an abrupt rise of AST, ALT and LDH levels within $12-24$ hours after the initiating event. After the treatment of the underlying IH causing condition, the AST level decreases toward normal faster than the ALT level. An increased Serum lactate level is also an important biomarker towards the detection of IH [13].

The current diagnosis method for IH results from the exclusion of all other possible causes of liver damage. At that point, the patient is treated by correction of the underlying condition that is causing IH [17]. This long process of exclusion leaves many cases untreated before the damage is too extensive. As a result, only $50 \%$ of hospital patients diagnosed with IH survive $[28,34,40,41]$. A better understanding of IH is needed to aid in diagnosis and treatment in order to improve the odds. Medical research is mostly limited to the data collected once patients are treated and data collected through experiments on rats and other small animals. A mathematical model can provide a new perspective and understanding of the condition. In present work, we develop a mathematical model to study the dynamics of $\mathrm{IH}$ with respect to systemic oxygen delivery in the body and measure the biomarkers associated with it, as IH is marked by massive increases of serum aminotransferase.

There are currently no mathematical models for ischaemic liver injury or hypoxic hepatitis. There are however, models of other types of liver injury, models of liver cell death, and models of biomarker production that helped us better understand and build this model [36]. The goals of this study are to: (i) provide a framework of relevant biomarkers and hepatocytes in order to study patterns of IH condition, (ii) estimate average decay and growth parameters related to concentration of biomarkers during IH, (iii) predict real time peak levels of different biomarkers, namely AST, ALT, and LDH, due to liver injury caused by IH at varied oxygen levels in the body as well as varied treatment frequency, and (iv) identify threshold conditions for time to reach the critical concentration level of hepatocytes for irreversible damage for varied initial oxygen level in the body. This study's framework involves building 
a mathematical model and focus on above goals. The model provides formulation of time to reach a state of $70 \%$ damaged hepatocyte (a critical condition of liver injury) starting from fixed oxygen level and from set initial state of liver under no treatment scenario. Once $70 \%$ of the liver's hepatocytes are damaged, the liver is unable to regenerate and a transplant may be necessary [29]. Knowing the time to reach a critical state of damaged hepatocyte will aid physicians to identify when there is an immediate need of liver transplant and allow a manipulation of drug treatment. This model also explores an estimate of level of further damage due to reperfusion. We validated our model results using data from $[4,10,18,40]$ that represents clinical conditions underlying hypoxic hepatitis.

We organize the paper as follows. Section 2 provides a detailed description of the mathematical model. Equilibrium points and stability analysis are discussed in Section 3. We also provide a sensitivity analysis of the equilibrium points with respect to an important parameter here. Numerical simulation of the mathematical model to predict the levels of bio-markers for a given value of delivered oxygen in the blood is presented in section 4. This model is also validated from data collected from literature in this section. We conclude with a discussion in Section 5.

\section{Methods}

\subsection{Data Sources}

We develop a functional form of changes in oxygen delivery in the body over time using empirical information from patient data. In this study, we used data corresponding to $50 \%$ initial oxygen level (the most common oxygen level observed in cases of ischaemic injury). The parameters describing the variations in AST, ALT, LDH are estimated using [20,34] (the definition of parameters are given in Table 1. The parameter describing the rate of oxygen return $\epsilon$ in Eq.7 is chosen based on the peak biomarker levels for $50 \%$ oxygen treated at 8 hours that closely matches the averages found in the literature $[20,34]$ (estimates of parameters are collected in Table 1.

In order to validate the model, a compiled data from four empirical studies on ischaemic liver injury $[4,10,18,40]$ are used. The relevant data, needed for fitting model results, are extracted to include only cases of ischaemic hepatitis due to cardiac injury or reduced oxygen. For example, the data for low $\mathrm{O}_{2}$ availability, low $\mathrm{O}_{2}$ delivery, and low $\mathrm{O}_{2}$ are collected from [4]. Similarly, from [18], only the data for acute cardiac failure and congestive heart failure are used for comparison. Since most biomarker data is collected at unknown intervals after ischaemic injury, the peak values are the best way to compare data. From the four papers $[4,10,18,40]$, an average peak serum value for AST ranging from 1927 - $4587 \mathrm{IU} / \mathrm{L}$, ALT ranging from 1803 - $1959 \mathrm{IU} / \mathrm{L}$, and LDH ranging from $3067-4494$ IU/L. Different data are used for parameter estimation and model validation. Model parameters are estimated using patient data from studies $[20,34]$.

\section{$\% \% \% \% \% \% \% \% \% \% \% \% \% \% \% \% \% \% \% \% \% \% \% \% \% \%$ \\ Fig. 1 \\ $\% \% \% \% \% \% \% \% \% \% \% \% \% \% \% \% \% \% \% \% \% \% \% \% \% \%$}

Validation of the model is carried out by comparing the model outputs with the corresponding quantities from data (such as peak biomarker values observed in hospitalized cases of IH due to cardiac injury or reduced oxygen; data obtained from $[4,10,18,40])$. These quantities are compared because currently, it is an effective clinical way to verify patient conditions, since the blood serum levels are standard checks for hospital patients. For AST, we have a maximum of $2681.95 \mathrm{IU} / \mathrm{L}$ with $50 \%$ oxygen availability in the body treated after 8 hours and around $3000 \mathrm{IU} / \mathrm{L}$ after $10 \mathrm{hrs}$ which fits in the range 1927 - $4587 \mathrm{IU} / \mathrm{L}$. For ALT, we have a maximum level of $1316.89 \mathrm{IU} / \mathrm{L}$ with $50 \%$ oxygen availability in the body treated after 8 hours and 1500 IU/L after 10 hours which falls short of the range 1803-1959 $\mathrm{IU} / \mathrm{L}$, and LDH with $50 \%$ oxygen availability in the body treated after 8 hours has a maximum of $3547.93 \mathrm{IU} / \mathrm{L}$ and around $3900 \mathrm{IU} / \mathrm{L}$ treated after 10 hours which fits in the range $3067-4494 \mathrm{IU} / \mathrm{L}$ in the literature (see Fig. 1). Most of these values are well within the overall ranges listed in the literature. The larger literature averages specially for ALT could possibly be due to data taken from 
more patients treated much later than 8 hours or starting with less than $50 \%$ oxygen. Other error could have come from a difference in the number of patients whose data are analyzed to gather the averages in each paper.

\subsection{Model Description}

We model hepatocyte death over time and focus on cell death due to depletion of adenosine triphosphate ATP(A) in the liver cells. Cells use energy source from ATP molecules and die due to dearth of it as it lacks the energy to carry out essential functions. This form of cell death is called necrosis (see $[12,27,32])$. To model this form of cell death we track the production and consumption of ATP. The majority of ATP in the human body is produced and consumed locally, as a result, our model assumes that all ATP production relevant to the liver is produced in the liver. Any ATP produced outside the liver is considered negligible here. Additionally, the majority of ATP production requires oxygen in a process called cellular respiration. This process provides $95 \%$ of the ATP in each cell $[14,39]$. When there is a lack of oxygen being delivered to the liver, it fails to produce enough ATP, resulting necrosis [32].

Since the production of ATP requires oxygen, the amount of oxygen $(\mathrm{O})$ the liver receives determines how much or how little ATP can be produced and thus how many cell death occurs [14]. Our model assumes a starting value of oxygen which is then restored at a certain time of treatment. We use a logistic function to better fit the physiology as the liver takes time to regain oxygenated blood [33]. We assume here that if the initial oxygen present is $70 \%$ or less in the body, there are chances of liver injury due to Ischaemia. Also, if $70 \%$ of the hepatocyte is damaged due to lack of oxygen, the liver does not recover on its own and a liver transplant is needed for survival [29]. Since data corresponding to $50 \%$ initial oxygen level is mostly available in literature, we will focus our result based on $50 \%$ initial oxygen level in the body.

We also model the production of aspartate aminotransferase-AST (S), alanine aminotransferaseALT (L) and lactate dehydrogenase-LDH (D) which are all important bio-markers and byproducts of liver damage. The levels of these bio-markers can be observed via blood tests and are often used in determining the type or severity of liver injury [17]. AST and ALT behave in a similar fashion with both being produced at a certain rate based on damaged hepatocytes. AST is found in most major organs throughout the body and is released when any of the cells are damaged [8]. ALT is only found in the liver making it a major indicator of liver injury [8]. As a result, AST levels in the blood rise much more than ALT in cases of cardiac injury, because the heart also releases AST. This means that the more liver damage that happens over a certain amount of time the higher the production of these enzymes will be.

Lactate dehydrogenase (LDH) is produced in cases of liver damage. However, it has been noticed to be much higher in liver injury due to a lack of oxygen. This is because LDH is produced as back up for use when cells are not getting enough ATP [9]. LDH is part of the ATP production process. This enzyme is a catalyst for the reaction that converts pyruvate, $\mathrm{NADH}$, and Hydrogen into lactate and $\mathrm{NAD}+$, and back. In a healthy liver, most of the ATP is produced through a combination of pyruvate and oxygen.

In cases of low oxygen, such as in IH, the body compensates by producing more through an anaerobic process called glycolysis. This process uses NAD + to produce enough ATP to keep the liver functioning until oxygen is returned. The increased production of LDH speeds up the conversion of pyruvate and NADH into NAD+ and allows the production of a small amount of ATP [14]. This process results in an increase in lactate, also referred to as lactic acid. The resulting build up of lactate halts this production of $\mathrm{LDH}$, allowing levels to normalize [9]. Since this process is only meant as a temporary fix, the increase in LDH occurs rapidly in a matter of hours before capping off at its maximum and slowly decreasing [18]. Furthermore, the resulting ATP produced is minimal compared to that produced by the remaining hepatocytes [14]. For this reason, we considered ATP produced by LDH to be negligible in this model. 


\section{$\% \% \% \% \% \% \% \% \% \% \% \% \% \% \% \% \% \% \% \% \% \% \% \% \% \%$}

Fig. 2

$\% \% \% \% \% \% \% \% \% \% \% \% \% \% \% \% \% \% \% \% \% \% \% \% \% \%$

$$
\begin{aligned}
\frac{d A}{d t} & =\rho H O(t)-k H \frac{A}{A_{\text {norm }}} \\
\frac{d H}{d t} & =r H\left(1-\frac{H+Z}{H_{\max }}\right)-\eta H\left(1-\frac{A}{A_{\text {norm }}}\right)-f\left(O^{\prime}(t)\right) \\
\frac{d Z}{d t} & =\eta H\left(1-\frac{A}{A_{\text {norm }}}\right)-\delta_{Z} Z+f\left(O^{\prime}(t)\right) \\
\frac{d S}{d t} & =\frac{\delta_{Z} \beta_{S}}{\theta H_{\max }} Z-\delta_{S}\left(S-S_{\text {min }}\right) \\
\frac{d L}{d t} & =\frac{\delta_{Z} \beta_{L}}{\theta H_{\max }} Z-\delta_{L}\left(L-L_{\min }\right) \\
\frac{d D}{d t} & =\frac{H_{D} \beta_{D}}{\theta H_{\max }}(1-O(t))\left(1-\frac{D}{D_{\max }}\right)-\delta_{D}\left(D-D_{\min }\right)
\end{aligned}
$$

where

$$
\begin{gathered}
O(t)=\frac{1-O_{0}}{1+e^{-\epsilon(t-\tau)}}+O_{0} \\
f\left(O^{\prime}\right)=\left|\frac{d}{d t} O(t)\right|=\left|\frac{\epsilon\left(1-O_{0}\right) e^{-\epsilon(t-\tau)}}{\left(1+e^{-\epsilon(t-\tau)}\right)^{2}}\right|= \begin{cases}\frac{\epsilon\left(1-O_{0}\right) e^{-\epsilon(t-\tau)}}{\left(1+e^{-\epsilon(t-\tau)}\right)^{2}} & \text { if } O_{0} \leq 1 \\
\frac{\epsilon\left(O_{0}-1\right) e^{-\epsilon(t-\tau)}}{\left(1+e^{-\epsilon(t-\tau)}\right)^{2}} & \text { if } O_{0}>1\end{cases}
\end{gathered}
$$

The term $f\left(O^{\prime}(t)\right)$ is the term for the damage due to reperfusion given by Eq.8. Note that this term is zero before treatment. Also, the right hand side of the system explicitly depend on time(t). The first term $r H\left(1-\frac{H+Z}{H_{\max }}\right)$ of the healthy hepatocyte model controls the production of new healthy hepatocytes. This term is scaled by the hepatocyte growth rate $r$ and has a carrying capacity of that total number of hepatocytes. This implies that the regrowth of the liver is limited by the death of its cells. However, this condition is not permanent, as the damaged hepatocytes are removed from the liver over time making room for new cells to grow. The second term $\eta H\left(1-\frac{A}{A_{\text {norm }}}\right)$ controls the death of healthy hepatocytes. This term is controlled by the current amount of ATP as opposed to its normal level and is scaled by the death rate $\eta$. The damaged hepatocytes are created using the death term from the healthy hepatocyte function. In this function the death term $\eta H\left(1-\frac{A}{A_{\text {norm }}}\right)$ is added. This creates a one to one conversion from healthy hepatocytes to damaged hepatocytes. The second term $\delta_{Z} Z$ controls the rate at which damaged hepatocytes are flushed from the liver. It is based on the lysis rate $\delta_{Z}$. ATP is also broken into two terms: the production term $\rho H O$ and the consumption term $k H \frac{A}{A_{\text {norm }}}$. Both terms are based on the number of healthy hepatocytes $(\mathrm{H})$, as they are both producing and consuming the ATP in the liver. The consumption is capped by $\frac{A}{A_{\text {norm }}}$ to keep ATP from going negative which would not make physiological sense. The production, however, is controlled by the percentage of oxygen $(\mathrm{O})$. The production and consumption of ATP is controlled by the two rates $\rho$ and $\mathrm{k}$. In our model we assume that these two rates are equal. For our oxygen we use a logistic function in order to model the return of oxygen to the liver after treatment. 
The return of blood and oxygen to the liver following treatment is called reperfusion. Since the reperfusion of blood to the liver takes roughly 6 hours [33], we chose $\epsilon$ to be 20 here to match our peak biomarker levels for $50 \%$ oxygen treated at 8 hours that closely matches the averages from the literature $[20,34]$. Additionally, we augment $\tau$ by a certain amount to offset the curve in order to have the time of treatment be reflected by the beginning of the rise in oxygen. With the logistic function we used, $\tau$ controls the midpoint of the curve so for all figures $\tau$ is offset by $\frac{3}{24}$ ( 3 hours). The biomarkers AST and ALT are modeled the same way as in [35]. The first terms of these equations model the production of the biomarkers by the damaged hepatocytes $\mathrm{Z}$. This production is controlled by the lysis rate of damaged hepatocytes $\delta_{Z}$, the amount of blood in the human body $\theta$, the maximum number of hepatocytes $H_{\max }$ and the total amount of each biomarker in the liver $\beta_{S}$ and $\beta_{L}$. The second term is the removal of the biomarkers from the bloodstream at a rate given by $\delta_{S}$ or $\delta_{L}$ for AST and ALT respectively. The minimum observed value of the biomarkers are used to prevent the levels from ever dropping below their minimum. The LDH equation is similar to the other biomarkers, with growth depending on the maximum hepatocytes and the total amount of blood in the human body, with a growth rate of $\beta_{D}$ The first term differs from the other biomarkers in few ways where AST and ALT are produced by damaged hepatocytes, $\mathrm{LDH}$ is produced by the remaining healthy hepatocytes to help the liver and as such we remove the dependence of the damaged hepatocyte lysis rate. We also include the term $1-O$ to match the physiology [9]. This allows the $\mathrm{LDH}$ level to increase when oxygen is less than 1, i.e. when the liver is being damaged. We also include the term $\left(1-\frac{D}{D_{\max }}\right)$ to prevent the level of LDH from increasing beyond the maximum dictated by the physiology [9]. Similar to the other biomarkers, $\mathrm{LDH}$ is removed from the bloodstream at a rate $\delta_{D}$ and capped to not go below the minimum observed level $D_{\min }$. Most of the parameters in this model are obtained from existing literature as shown in Table 1. However, some of the parameters in our model are estimated to achieve physiologically reasonable behaviour.

\section{Analysis (a case when $O(t)$ is independent of t, i.e., $O$ is constant):}

\subsection{Steady State Analysis}

The steady states of $A, H, Z, S, L$, and $D$ are solved assuming a full oxygen percentage, or $O=1$ when is oxygen is independent of $t$ as shown in Fig. 3. The amount of oxygen, given in Eq. 7 reaches saturation after treatment and does not affect the equilibrium analysis. This reveals different steady states based on the relation of the ATP production and consumption rates, $\rho$ and $k$. The trivial case is $H^{*}=0, Z=H_{\max }, A^{*}=0$ and the bio-markers above the minimum which happens when a patient dies. We now consider the case when $H^{*}$ is nonzero. Here we create a new variable $\lambda$ such that $\lambda=\left(1-\frac{\rho}{k}\right)$. There exist the following steady states:

$$
\begin{aligned}
H^{*} & =H_{\max } \frac{1-\frac{\eta}{r} \lambda}{1+\frac{\eta}{\delta_{Z}} \lambda} \\
A^{*} & =A_{\text {norm }}(1-\lambda) \\
Z^{*} & =\frac{\eta \lambda}{r} H_{\max }\left[\frac{r-\eta \lambda}{\delta_{Z}+\eta \lambda}\right] \\
S^{*} & =\frac{\delta_{Z} \beta_{S} \eta \lambda}{r \delta_{S} \theta}\left[\frac{r-\eta \lambda}{\delta_{Z}+\eta \lambda}\right]+S_{\text {min }} \\
L^{*} & =\frac{\delta_{Z} \beta_{L} \eta \lambda}{r \delta_{L} \theta}\left[\frac{r-\eta \lambda}{\delta_{Z}+\eta \lambda}\right]+L_{\text {min }} \\
D^{*} & =D_{\min }
\end{aligned}
$$


These equations reveal restrictions on the possible values of $\lambda$. The ideal case is when $\lambda=0$, which happens when $\rho=k$. In this case we get the following steady states from the above equations:

$$
\begin{aligned}
H^{*} & =H_{\max } \\
A^{*} & =A_{\text {norm }} \\
Z^{*} & =0 \\
S^{*} & =S_{\min } \\
L^{*} & =L_{\min } \\
D^{*} & =D_{\min }
\end{aligned}
$$

This case is for our model assumption. Since oxygen is set to be full in the steady state, the patient would be healthy or treated when this steady state occurs. A healthy patient have hepatocyte levels $H$ returned to a maximum, resulting in zero damaged hepatocytes $Z$. This would also result in normal levels of ATP and the biomarkers AST, ALT, and LDH which are the normal/minimum values listed above. It is for this reason that the value of $\rho$ is assumed to be equal to $k$ in our model.

In the cases where $\rho \neq k$ we run into steady states that are physically impossible. We examine two possibilities,

- Consider, $\lambda<0$. When $\lambda$ is negative, as is the case where $\frac{\rho}{k}>1$, a negative steady state for the damaged hepatocytes $Z^{*}$ can occur. This is the case where $\frac{\delta_{Z}}{\eta}>|\lambda|$. A negative $Z$ value corresponds to a negative amount of damaged hepatocytes and an amount of healthy hepatocytes greater than the amount of hepatocytes in the liver which cannot realistically occur. Similarly, if $\frac{\delta_{Z}}{\eta}<|\lambda|$ the steady state of the healthy hepatocytes is negative. This negative state would not only imply that the patient is dead, but also that they had more damaged hepatocytes than the total number of hepatocytes they started with. Analysis of the remaining case where $\frac{\delta_{Z}}{\eta}=|\lambda|$ revealed the trivial steady state where there are no healthy nor damaged hepatocytes present. The failure of all possible relations between $\lambda$ and $\frac{\delta_{Z}}{\eta}$ when $\lambda<0$ prove that $\lambda$ must always be positive.

- Consider, $\lambda>0$. The possibility for $\lambda$ to be positive also fails under certain conditions. Both the healthy and damaged hepatocytes would be negative if $\frac{r}{\eta}<\lambda$ and zero if $\frac{r}{\eta}=\lambda$. Therefore, the only physically possible steady state that occurs when $\rho \neq k$ is that where $\frac{\rho}{k}<1$ and $\frac{r}{\eta}>1-\frac{\rho}{k}$.

These resulting steady states however, do not return to normal values expected of a healthy patient. This indicates that there is still an underlying condition that is damaging the liver. Since we are assuming treatment at this point, this too is an unrealistic condition for our model. We determine the stability of the steady states by analyzing the Jacobian of the system of differential equation. Linearizing the system of intracellular and hepatocyte differential equations yields the Jacobian

$$
J=\left[\begin{array}{cccccc}
-\frac{k H}{A_{\text {norm }}} & \rho O-\frac{k A}{A_{\text {norm }}} & 0 & 0 & 0 & 0 \\
\frac{\eta H^{\prime}}{A_{\text {norm }}} & r\left(1-\frac{H+Z}{H_{\text {max }}}\right)-\frac{r H}{H_{\text {max }}}-\eta\left(1-\frac{A}{A_{\text {norm }}}\right) & -\frac{r H}{H_{\max }} & 0 & 0 & 0 \\
-\frac{\eta H}{A_{\text {norm }}} & \eta\left(1-\frac{A^{*}}{A_{\text {norm }}}\right) & -\delta_{Z} & 0 & 0 & 0 \\
0 & 0 & \frac{\delta_{z} \beta_{S}}{\theta H_{\text {max }}} & -\delta_{S} & 0 & 0 \\
0 & 0 & \frac{\delta_{z} \beta_{L}}{\theta H_{\max }} & 0 & -\delta_{L} & 0 \\
0 & \frac{\beta_{D}}{\theta H_{\max }}(1-O)\left(1-\frac{D}{D_{\max }}\right) & 0 & 0 & 0 & -\frac{H \beta_{D}}{\theta H_{\max }}\left(\frac{(1-O)}{D_{\max }}\right)-\delta_{D}
\end{array}\right]
$$

The system has unique steady state that is numerically seen to be locally stable. In particular, corresponding to the equilibrium points Eq.10 the eigenvalues corresponding to the Jacobian are $-\delta L$, -r, $-\delta S,-\delta Z,\left(-k * H_{\max }\right) /$ Anorm, $-\delta D$. This healthy equilibria characterized by Eq.10 is locally stable. 


\subsection{Variation in the Equilibrium with Respect to $\rho$ and $k$.}

We now discuss the sensitivity of the equilibrium points, in other words we determine how perturbations to the parameter $\rho$ and $\mathrm{k}$ affect the equilibrium points $H^{*}, Z^{*}, Z^{*}, S^{*}$ and $L^{*}$ [7]. Specifically we calculate the normal sensitivity index of $H^{*}$ with respect to $\rho$ [7] given by $S I\left[H^{*} ; \rho\right]=$ $-\frac{\eta k \rho\left(\delta_{Z}+r\right)}{\left(k\left(\delta_{Z}+\eta\right)-\eta \rho\right)(k(\eta-r)-\eta \rho)}$. Similarly we calculate the normal sensitivity index of the other variables as shown in [7] with respect to $\rho$ and $\mathrm{k}$. The magnitude of sensitivity index outlines the relative importance of that variable with respect to equilibrium and the sign represents either proportional or reciprocal relationship. We plot the sensitivity index of these variables using Mathematica in Fig. 4. As seen in Fig. 4, increasing $k$ by $1 \%$ yields an approximate $3.3 \%$ increase in $Z^{*}, S^{*}, L^{*}$. All these three equilibrium points have almost equal impact on $k$. Similarly a $1.8 \%$ increase occurs in $H^{*}$. This implies that $Z^{*}, S^{*}, L^{*}$ have higher sensitivity compared to $H^{*}$. Also, $Z^{*}, S^{*}, L^{*}$ has negative sensitivity index with respect to $\rho$ which implies if $\rho$ decreases then the equilibrium point increases.

\section{$\% \% \% \% \% \% \% \% \% \% \% \% \% \% \% \% \% \% \% \% \% \% \% \% \% \%$ \\ Fig. 4 \\ $\% \% \% \% \% \% \% \% \% \% \% \% \% \% \% \% \% \% \% \% \% \% \% \% \% \%$}

\section{Numerical Results (a case when $O(t)$ is explicit function of $\mathbf{t}$ ):}

We consider three scenarios here how biomarkers changes with time as treatment $\tau$, how biomarkers changes with initial $O_{0}$ level present in the body, how biomarkers changes with and without treatment and what is the rate of irreversible damage with changes in initial oxygen $O_{0}$. Here we have considered oxygen $O(t)$ as explicit functions of time t. We numerically solve the system of equations 1-6 using ode solver in Python. Results are generated by initializing a certain time of treatment and then varying the initial oxygen level. The lower the initial oxygen level, the more severe the damage to the liver will be.

The liver is able to regrow even after sustaining an incredible amount of damage, however there is a threshold from which a liver cannot regrow itself. This threshold is roughly around $30 \%$ of healthy hepatocytes [29] but it can vary slightly from individual to individual. Thus, any patient with liver damage over $70 \%$ may be at risk of liver failure and is most likely in need of a liver transplant [29]. This critical point is indicated by a dotted line on the graphs Fig. 8 of hepatocytes. In any instances where the hepatocyte curve dips below this line, critical damage is sustained.

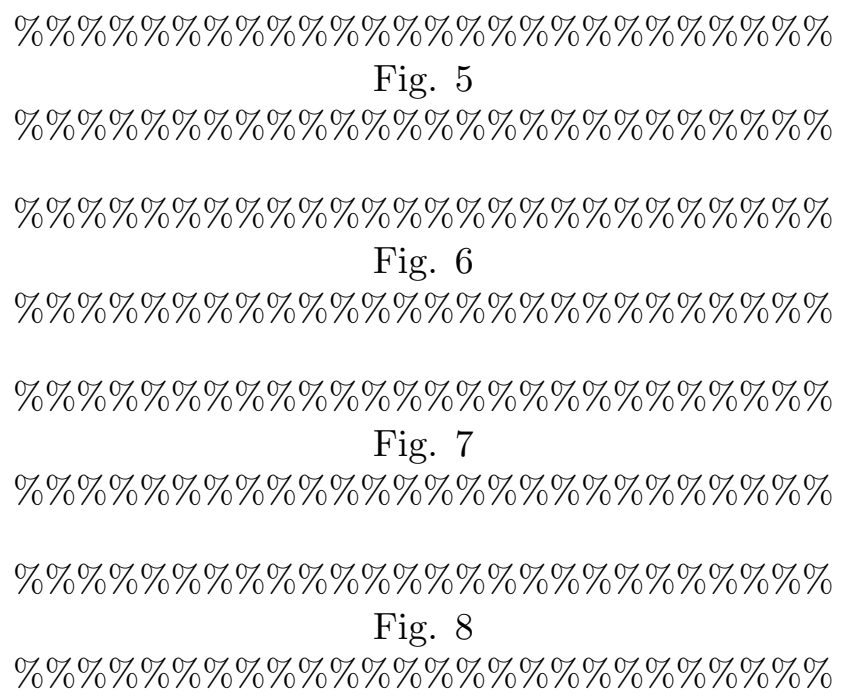




\subsection{Hepatocyte Damage at Varied Treatment Frequency}

Figure 5b, 6b, 7b, 8b shows the plot of ALT, AST, LDH and Hepatocyte values varied at different oxygen levels treated after eight hours. The increased severity of hepatocyte damage can also be observed through a sharper rise in AST, ALT and LDH. In this case AST spikes faster than ALT and also returns to its normal value more rapidly while ALT takes over a week to return to its normal value. These observed slope changes and normalization times appear to be similar to what is observed with experimental data [18]. LDH also spikes very quickly, however it gets capped off at an upper limit. This is because LDH production in the liver slows as LDH builds up eventually hitting a certain limit at which point the body ceases LDH production [9].

\subsection{Hepatocyte Damage at Varied Levels of Initial Oxygen}

Plots at Fig. 5a, Fig. 6a, Fig. 7a, Fig. 8a are generated by taking a common initial oxygen level in cases of IH and varying the time of treatment. The common level selected from the literature is 50\% [18]. As expected, the longer the oxygen is left below its normal value, the more the liver is damaged. Since the oxygen level is the same across all treatment times, the damage happens at the same rate up until the time of treatment.

\subsection{Critical Threshold of Damaged Hepatocytes for Liver Transplant}

We observed in Fig. 8b that at almost $40 \%$ oxygen the liver will reach critical damage if treated at or after 8 hours. If treated sooner, the patient will be less likely to need a liver transplant. Due to the return of oxygen over a period of $6 \mathrm{hrs}$, there is still damage for a while after treatment is administered [33]. This correlates to the space between the treatment and the time of the trough observed in this figure. The associated AST and ALT graphs show similar results with the peaks also occurring at time after treatment and increasing with prolonged untreated time.

Interestingly, when there is no treatment given AST, ALT and LDH spikes and begins to decay, despite the fact that liver cell death is still occurring. This is because the number of damaged hepatocytes are removed through a process called lysis [36]. Since AST and ALT are directly related to the amount of damaged hepatocytes, this explains the decay while there being no treatment. LDH, on the other-hand, returns to normal due to the fact that it is produced by healthy hepatocytes [9]. With no treatment being administered the number of healthy hepatocytes eventually dwindles to zero and LDH, AST, ALT production ceases. Through Fig.9a our model shows different time scenarios of hepatocyte damage beyond repair for varied oxygen levels when there is no treatment. This means that with a lower initial oxygen level, the time before liver damage becomes severe (dropping below $30 \%$ remaining healthy hepatocytes) is shorter than if the initial oxygen level is higher. Fig. 9b shows the estimated timing of reaching the critical $30 \%$ hepatocyte level for irreversible damage for different initial $O_{2}$ level. The scatter plot fits the exponent function $f(x)=a e^{k x}+c$, where $a=2.68176\left(10^{-8}\right)$, $k=0.216333$ and $c=0.314243$ are estimated using Mathematica. The model also helps estimate a time frame for treatment as shown in Fig. 9a. Thus this plot may help physician to estimate the time of irreversible damage given different levels of initial oxygen avaliability in patients. It is also observed in Fig. 8a, Fig. 8b that hepatocytes begin to recover shortly after treatment is administered which indicates that this model can aid physician estimate the time for treatment.

$$
\begin{aligned}
& \text { \%\%\%\%\%\%\%\%\%\%\%\%\%\%\%\%\%\%\%\%\%\%\%\%\%\% } \\
& \text { Fig. } 9 \\
& \text { \%\%\%\%\%\%\%\%\%\%\%\%\%\%\%\%\%\%\%\%\%\%\%\%\%\% }
\end{aligned}
$$

\subsection{Ischaemia-reperfusion Injury}

Ischaemia-reperfusion injury (IRI) or reperfusion injury or reoxygenation injury, is the tissue damage caused when blood supply returns to tissue after a period of ischaemia or lack of oxygen (anoxia or hypoxia). The absence of oxygen and nutrients from blood during the ischaemic period creates a condition in which the restoration of circulation results in inflammation which leads to the death of the 
cells and oxidative damage through the induction of oxidative stress rather than restoration of normal function [33]. There is a sizable amount of cell death that happens when the liver is reperfused with oxygenated blood [23]. Current sources disagree on how much of the damage a liver with IH sustains is from reperfusion and how much is from necrosis $[8,18,33]$. Because of this, we model damage due to reperfusion separately from our main model.

In order to model the damage due to reperfusion we use the derivative of our oxygen function. In this case we have $f\left(O^{\prime}(t)\right)=\left|\frac{d O}{d t}\right|$. This is because the damage during reperfusion is caused by the rapid influx of oxygenated blood [33]. By using the absolute value of the derivative, we take into account equal damage due to the rate of oxygen decrease and increase. This accounts for any damage that occurs due to the sudden loss of oxygen when IH occurs, as well as reperfusion damage since both cases cause the mass amount of inflammation leading to cell death [33]. Since this term is damage to the liver, it is taken away from healthy hepatocytes and added to damaged hepatocytes.

The absolute value is multiplied by a variable that we call the reperfusion rate c. With units of cells/day the value of $\mathrm{c}$ is the rate at which hepatocytes die due to reperfusion damage. Through our research of reperfusion injury, we are unable to determine this rate without further data or reconstruction of our oxygen equation. Reperfusion also creates organ damage following transplantation due to the return of oxygenated blood. As a result, much of the literature we obtain on the topic of reperfusion focuses on transplant cases where different organs are completely reperfused over periods of time longer than expected in our case $[6,46]$. This leads us to estimate the values of $\mathrm{c}$ for the model. We assume that the damage due to reperfusion do not exceed that due to ischaemia. Therefore, we select values of $c$ that produces less damage than our original model. Trial of various $c$ values reveals that values larger than $\mathrm{c}=2$ produces damage comparable to our ischaemia model. Therefore, we estimate a c value between 0 and 2 cells/day.

This yields the model for our new healthy hepatocyte function and our new damaged hepatocyte function.

$$
\begin{aligned}
& \frac{d H}{d t}=r H\left(1-\frac{H+Z}{H_{\max }}\right)-\eta H\left(1-\frac{A}{A_{\text {norm }}}\right)-c\left|\frac{d O}{d t}\right| \\
& \frac{d Z}{d t}=\eta H\left(1-\frac{A}{A_{\text {norm }}}\right)-\delta_{Z} Z+c\left|\frac{d O}{d t}\right| \text {. } \\
& \text { \%\%\%\%\%\%\%\%\%\%\%\%\%\%\%\%\%\%\%\%\%\%\%\%\%\% } \\
& \text { Fig. } 10 \\
& \text { \%\%\%\%\%\%\%\%\%\%\%\%\%\%\%\%\%\%\%\%\%\%\%\%\%\% }
\end{aligned}
$$

Adding reperfusion damage, the model causes a large loss of cells immediately following treatment which means that at the time of treatment there is still a sizable amount of cell death that occurs. This additional cell death means that the danger threshold of liver damage before treatment is higher than the previously discussed $30 \%$. It takes into account the possible damage from reperfusion and finds values where the liver would still maintain $30 \%$ of its healthy hepatocytes after reperfusion.

\section{Discussion}

Ischemic Hepatitis $(\mathrm{IH})$ is a dangerous liver injury with a 50\% mortality rate [40]. Cases of IH are caused by an underlying medical condition such as cardiac injury, respiratory injury, sepsis, or other types of shock. The underlying condition results in a decrease in the amount of oxygenated blood available to the liver, often greater decrease than can be mediated by the liver's natural hepatic arterial buffer response [18]. Without adequate oxygen, the liver cells (i.e., hepatocytes) cannot produce sufficient ATP, the cell's primary energy source. A reduction in ATP results in hepatocyte death through a process called necrosis. Extensive necrosis results in irreversible liver damage. The death of hepatocytes are identified by significant spikes in the blood serums such as AST, ALT, and LDH. Despite the severity of this condition, IH is limited to diagnosis via exclusion of other liver injuries and then treatment of the underlying cause. However, to best of our knowledge, there has not 
been any systematic dynamic study that attempts to understand the role of biomarkers and oxygen level on the concentration of healthy and damaged hepatocytes [36]. Hence, it is critical to develop an approach and a dynamic framework that may provide a reference for physicians to diagnose cases of IH and determine whether or not patients have reached critical damage based on their blood serum levels on real time basis.

In this study, we develop and analyze a novel mathematical model that capture a coupled dynamics of hepatocytes, cells death due to ischemic hepatitis and relevant biomarkers (AST, ALT, and LDH). The derived model is based upon decay and regeneration of healthy and damaged hepatocytes in the liver. In order to model IH condition via dynamics of hepatocytes several assumptions are considered to understand the systematic damage of the hepatocytes. Due to the range of conditions that result in $\mathrm{IH}$, we focus solely on cardiac injury. We assume that the cardiac injury decreases blood flow to the liver that results in a lack of oxygen. It is also assumed that any hepatocyte death is the result of necrosis due to insufficient ATP. This excludes hepatocyte death by any other processes. We consider that the hepatocytes produce and consume ATP at the same rate. We also assume that any affect to ATP levels as a result of the LDH serum levels is minimal and does not effect the results of the model. The resulting model describes the death of the liver's hepatocytes, the reduction of ATP, and the resulting biomarker spikes due to a logarithmic oxygen function. This model is presented by a system of six differential equations. The biomarkers AST, ALT, and LDH are then compared with patient data from various studies $[4,20]$ and model results are validated. This comparison allows us to verify that the resulting biomarker levels are close to what is actually observed in cases of IH. Later model is modified to include additional hepatocyte damage due to the return of oxygenated blood to the liver in the process known as reperfusion. This component is added to help predict damage due to treatment and aid in the development of better treatment methods.

The model shows the effects of treatment time and oxygen level due to initial injury on the extent of hepatocyte death and the levels of the biomarkers. We observe that a lower initial oxygen percent results in greater cell death, as is expected. For example, it is seen in Figure 9a that with no treatment and $30 \%$ initial oxygen it takes approximately 12 hours for $70 \%$ of the hepatocytes to die. However, with $80 \%$ initial oxygen, it takes closer to 36 hours to reach the same percentage. This range shows that it takes between 12 and 36 hours for ischemic liver injury to become irreversible. Similarly, we observe that more cell death occurred with a delay in treatment, correlating with higher biomarker levels. It is observed that LDH spikes the quickest and takes the longest to return to normal as compared to AST and ALT. AST spikes at similar rate to LDH but returns to normal much more quickly. The biomarker ALT spikes slower than the other two but returns to normal at a rate similar to that of LDH. As can be seen in Figure 9b, the biomarkers will return to normal despite a lack of treatment due to their natural flushing from the blood. In summary, the initial level of oxygen and time to treatment since patient hospitalized is crucial to predict peak levels of biomarkers and hence, to estimate the level of liver damage.

As seen in Fig.8a, the treatment time is a crucial factor in determining the extent of liver damage. By delaying treatment by just 4 hours the damage extends from $40 \%$ to over $60 \%$ for the most common initial oxygen. Given the initial oxygen percent in the body, a physician can estimate the time since injury and diagnosis by comparing the peak levels of patient's AST, ALT, and LDH levels upon diagnosis as displayed in Fig. 5a, 6a, and 7a. Similarly, if the approximate time of injury is known, comparing a patient's peak biomarker levels as in Fig. 7b, Fig. 6b, and Fig. 5b, physicians can estimate the percent oxygen present in body after damage. Thus the information of initial oxygen percent, combined with the knowledge of treatment time is useful to predict if the patient's liver damage has passed the critical 70\% damage threshold for transplant, expediting the treatment process [29]. In the case, the threshold has likely been passed, the physician should start to look into the possibility of liver transplant and other forms of treatment.

In spite of some practical clinical results from this modeling study, there are some limitations. We consider only one form of cell death and further refined modeling work can include the other form of cell death referred as apoptosis. Apoptosis is the process by which cells break apart as a result of biochemical reactions with cytokines that are released by the cells themselves [32]. A better understanding and model of apoptosis will help improve our reperfusion model. The reperfusion of 
oxygen into the liver produces inflammation and a release of tumor necrosis factor alpha (TNF- $\alpha$ ), a cytokine that can result in cell death via apoptosis [32] [38]. It would be ideal to find a rate that is slow enough to reduce reperfusion damage but quick enough to prevent irreversible damage due to ischaemia. Additional biomarker can be added to our existing model in order to generate spectrum of clinical conditions observed in patients. This can include bilirubin which observes a moderate spike in cases of IH, or the blood's clotting factor. The clotting factor is related to the INR level, another common blood measurement for which many of our sources contains data. In spite of these limitations, this study provides a first starting point where systematic mechanisms for IH can be identified and thoroughly evaluated. However, since new parameters are described here to study the dynamics of hepatocytes, there is strong need for relevant data, which is currently missing from empirical literature, to parameterize the model. 


\section{References}

[1] Aboelsoud, M. M., Javaid, A. I., Al-Qadi, M. O., And Lewis, J. H. Hypoxic hepatitis - its biochemical profile, causes and risk factors of mortality in critically-ill patients: a cohort study of 565 patients. Journal of critical care 41 (2017), 9-15.

[2] Berendsen, T., Izamis, M., Xu, H., Liu, Q., Hertl, M., Berthiaume, F., Yarmush, M., AND UYGUn, K. Hepatocyte viability and adenosine triphosphate content decrease linearly over time during conventional cold storage of rat liver grafts. In Transplantation proceedings (2011), vol. 43, Elsevier, pp. 1484-1488.

[3] Bergman, J. Atp: the perfect energy currency for the cell. Creation Research Society Quarterly 36, 1 (1999), 2-9.

[4] Birrer, R., Yasuharu, T., And Tsuyoshi, T. Hypoxic hepatopathy: Pathophysiology and prognosis. Internal Medicine 46, 14 (2007), 1063-70.

[5] Buil-Bruna, N., López-Picazo, J.-M., Moreno-Jiménez, M., Martín-Algarra, S., Ribba, B., AND Trocóniz, I. F. A population pharmacodynamic model for lactate dehydrogenase and neuron specific enolase to predict tumor progression in small cell lung cancer patients. The AAPS journal 16, 3 (2014), 609-619.

[6] Cannistrá, R., Zullo, G., Serafini, M., Naso, G., and Serra, N. Hepatic ischemia reperfusion injury: A systematic review of literature and the role of current drugs and biomarkers. International Journal of Surgery 33, 1 (2016), S57-S70.

[7] Chowell, G., Hyman, J. M., Bettencourt, L. M., Castillo-Chavez, C., and Nishiura, H. Mathematical and statistical estimation approaches in epidemiology. Springer, 2009.

[8] CIOBAnU, A. O., AND GHERASIM, L. Ischemic hepatitis-intercorrelated pathology. Maedica 13, 1 (2018), 5 .

[9] Corner, E., Geis, I., And Goodsell, D. Lactate dehydrogenase.

[10] Drolz, A., Horvatits, T., Michl, B., Roedl, K., Schellongowski, P., Holzinger, U., Zauner, C., Heinz, G., Madl, C., Trauner, M., and Fuhrmann, V. Statin therapy is associated with reduced incidence of hypoxic hepatitis in critically ill patients. Journal of Hepatology 60, 6 (2014), 1187-1193.

[11] Ebert, E. C. Hypoxic liver injury. In Mayo Clinic Proceedings (2006), vol. 81, Elsevier, pp. 12321236.

[12] Eguchi, Y., Shimizu, S., And Tsujimoto, Y. Intracellular atp levels determine cell death fate by apoptosis or necrosis. Cancer research 57, 10 (1997), 1835-1840.

[13] Fuhrmann, V., JÄGer, B., Zubkova, A., And Drolz, A. Hypoxic hepatitis-epidemiology, pathophysiology and clinical management. Wiener Klinische Wochenschrift 122, 5-6 (2010), 129139.

[14] Gnaiger, E., Steinlechner-Maran, R., Méndez, G., Eberl, T., and Margreiter, R. Control of mitochondrial and cellular respiration by oxygen. Journal of bioenergetics and biomembranes 27, 6 (1995), 583-596.

[15] Gray-Wilson, N. Douglas wilkin, ph. d.

[16] Gunn, V. L., And Nechyba, C. The harriet lane handbook: a manual for pediatric house officers. In The Harriet Lane handbook: a manual for pediatric house officers. Mosby, 2000.

[17] Hall, M. A. Ischemic hepatitis: Risks for sudden liver dysfunction. The Journal for Nurse Practitioners 13, 5 (2017), e253-e254. 
[18] Henrion, J. Hypoxic hepatitis. Liver International 32, 7 (2012), 1039-1052.

[19] Henrion, J., De, S. M., Schapira, M., Ghilain, J., Maisin, J., Gerard, R., and Heller, F. Hypoxic hepatitis: a difficult diagnosis when the cardiomyopathy remains unrecognized and the course of liver enzymes follows an atypical pattern. a report of two cases. Acta gastro-enterologica Belgica 61, 3 (1998), 385-389.

[20] Henrion, J., Schapira, M., Lumaert, R., Colin, L., Delannoy, A., and Heller, F. Hypoxic hepatitis: Clinical and hemodynamic study in 142 consecutive cases. Medicine 82, 6 (2003), 392-406.

[21] Hickman, P. E., And Potter, J. M. Mortality associated with ischaemic hepatitis. Australian and New Zealand journal of medicine 20, 1 (1990), 32-34.

[22] Johnson, R., O'Connor, M. L., And Kerr, R. M. Extreme serum elevations of aspartate aminotransferase. American Journal of Gastroenterology 90, 8 (1995).

[23] Kalogeris, Baines, K.-A. K. Cell biology of ischemia/reperfusion injury. International Review of Cell and Molecular Biology 298 (2012), 229-317.

[24] Kanel, G. C., Ucci, A. A., Kaplan, M. M., and Wolfe, H. J. A distinctive perivenular hepatic lesion associated with heart failure. American journal of clinical pathology 73, 2 (1980), $235-239$.

[25] Kavoliuniene, A., Vaitiekiene, A., and Cesnaite, G. Congestive hepatopathy and hypoxic hepatitis in heart failure: a cardiologist's point of view. International journal of cardiology 166, 3 (2013), 554-558.

[26] KILliP III, T., And PAyne, M. A. High serum transaminase activity in heart disease: circulatory failure and hepatic necrosis. Circulation 21, 5 (1960), 646-660.

[27] Leist, M., Single, B., Castoldi, A. F., Kühnle, S., And Nicotera, P. Intracellular adenosine triphosphate (atp) concentration: a switch in the decision between apoptosis and necrosis. Journal of Experimental Medicine 185, 8 (1997), 1481-1486.

[28] Lightsey, J. M., And Rockey, D. C. Current concepts in ischemic hepatitis. Current opinion in gastroenterology 33, 3 (2017), 158-163.

[29] Michalopoulos, G. K., And DeFrances, M. C. Liver regeneration. Science 276, 5309 (1997), $60-66$.

[30] Møller, S., And Bernardi, M. Interactions of the heart and the liver. European heart journal 34, 36 (2013), 2804-2811.

[31] Myers, J., And Hickam, J. An estimation of the hepatic blood flow and splanchnic oxygen consumption in heart failure. The Journal of clinical investigation 27, 5 (1948), 620-627.

[32] Nanji, A. A., And Hiller-Sturmhöfel, S. Apoptosis and necrosis. Alcohol Health Res World 21, 4 (1997), 325-30.

[33] Pannen, B. H. New insights into the regulation of hepatic blood flow after ischemia and reperfusion. Anesthesia \& Analgesia 94, 6 (2002), 1448-1457.

[34] Raurich, J. M., Llompart-Pou, J. A., Ferreruela, M., Colomar, A., Molina, M., Royo, C., Ayestarán, I., And IbÁÑez, J. Hypoxic hepatitis in critically ill patients: incidence, etiology and risk factors for mortality. Journal of Anesthesia 25, 1 (2011), 50-56.

[35] Remien, C. H., Adler, F. R., Waddoups, L., Box, T. D., and Sussman, N. L. Mathematical modeling of liver injury and dysfunction after acetaminophen overdose: early discrimination between survival and death. Hepatology 56, 2 (2012), 727-734. 
[36] Remien, C. H., Sussman, N. L., And Adler, F. R. Mathematical modelling of chronic acetaminophen metabolism and liver injury. Mathematical medicine and biology: a journal of the IMA 31, 3 (2013), 302-317.

[37] Seeto, R. K., Fenn, B., And Rockey, D. C. Ischemic hepatitis: clinical presentation and pathogenesis. The American journal of medicine 109, 2 (2000), 109-113.

[38] Shuh, M., Bohorquez, H., Loss, G., And Cohen, A. Tumor necrosis factor- $\alpha$ : Life and death of hepatocytes during liver ischemia/reperfusion injury. The Oschner Journal 13, 1 (2013), $119-130$.

[39] Steinlechner-Maran, R., Eberl, T., Kunc, M., Margreiter, R., and Gnaiger, E. Oxygen dependence of respiration in coupled and uncoupled endothelial cells. American Journal of Physiology-Cell Physiology 271, 6 (1996), C2053-C2061.

[40] Tapper, E. B., Sengupta, N., And Bonder, A. The incidence and outcomes of ischemic hepatitis: a systematic review with meta-analysis. The American journal of medicine 128, 12 (2015), 1314-1321.

[41] Taylor, R. M., Tujios, S., Jinjuvadia, K., Davern, T., Shaikh, O. S., Han, S., Chung, R. T., Lee, W. M., And Fontana, R. J. Short and long-term outcomes in patients with acute liver failure due to ischemic hepatitis. Digestive diseases and sciences 57, 3 (2012), 777-785.

[42] Trilok, G., Qing, Y. C., And Li-Jun, X. Hypoxic hepatitis: a challenging diagnosis. Hepatology international 6, 4 (2012), 663-669.

[43] Waseem, N., And Chen, P.-H. Hypoxic hepatitis: a review and clinical update. Journal of clinical and translational hepatology 4, 3 (2016), 263.

[44] Weisberg, I. S., and Jacobson, I. M. Cardiovascular diseases and the liver. Clinics in liver disease 15, 1 (2011), 1-20.

[45] Whitehead, M., Hawkes, N., Hainsworth, I., And Kingham, J. A prospective study of the causes of notably raised aspartate aminotransferase of liver origin. Gut 45, 1 (1999), 129-133.

[46] Zhai, P., Hong, B., And Kupiec-Weglinski. Ischaemia-reperfusion injury in liver transplantation - from bench to bedside. Nat Rev Gastroenterol Hepatol 10, 2 (2012), 79-89. 


\section{List of Figures and Captions:}

1. Fig. 1: Bio-marker data collected from literature to include cases of ischaemic hepatitis due to low $\mathrm{O}_{2}$ availability, low $\mathrm{O}_{2}$ delivery. The (mean, standard deviation) IU/L of AST, ALT, LDH are $(2877.0, \sqrt{868.2}),(1821.4, \sqrt{266.6}),(4273.8, \sqrt{1486.9})$ respectively.

2. Fig. 2: Schematic depiction of the model. Solid arrow indicates conversion of the same variable from one state to another, dotted arrow indicates influence of a variable on the transition rate, and double arrow indicates secretion of biomarkers. See expressions terms of the Model System 1-6 to understand the appropriateness of arrows.

3. Fig. 3: Demonstration of the oxygen function at $50 \%$ initial $O_{0}$ values for different values of $\epsilon$.

4. Fig. 4: Sensitivity Indexes of the equilibrium concentration of variables with respect to parameters $\rho$ and $k$. Note, $\rho$ negatively and $k$ positively impacts equilibrium concentration of all model variables.

- Fig. 5a: ALT peaks with varied times of treatment at $50 \%$ oxygen level.

- Fig. 5b: ALT peaks with varied initial oxygen levels treated after 8 hours.

5. Fig. 6: AST peaks with varied times of treatment and oxygen levels.

- Fig. 6a: AST peaks with varied times of treatment at $50 \%$ oxygen level.

- Fig. 6b: AST peaks with varied initial oxygen levels treated after 8 hours.

6. Fig. 7: LDH peaks with varied times of treatment and oxygen levels.

- Fig. 7a: LDH peaks with varied times of treatment at $50 \%$ oxygen level.

- Fig. 7b: LDH peaks with varied initial oxygen levels treated after 8 hours.

7. Fig. 8: Hepatocyte damage with varied times of treatment and oxygen levels.

- Fig. 8a: Healthy Hepatocytes as function of normal with varied times of treatment at 50 $\%$ oxygen level.

- Fig. 8b: Healthy Hepatocytes as a function of normal with varied initial oxygen levels treated after 8 hours.

8. Fig. 9: Demonstration of the Hepatocyte, AST, ALT, LDH level with varied O2 levels when there is no treatment.

- Fig. 9a: Hepatocyte death with varied O2 levels when there is no treatment.

- Fig. 9b: The estimated time of reaching the critical 30\% Hepatocyte level for irreversible damage given the initial oxygen level.

9. Fig. 10: Hepatocyte damage with varied rates of reperfusion treated at the 8 th hour.

\section{List of Tables and Captions:}

1. Table. 1: Table of Variables. 
bioRxiv preprint doi: https://doi.org/10.1101/2020.03.11.987594; this version posted March 11, 2020. The copyright holder for this preprint (which was not certified by peer review) is the author/funder, who has granted bioRxiv a license to display the preprint in perpetuity. It is made available under aCC-BY 4.0 International license.

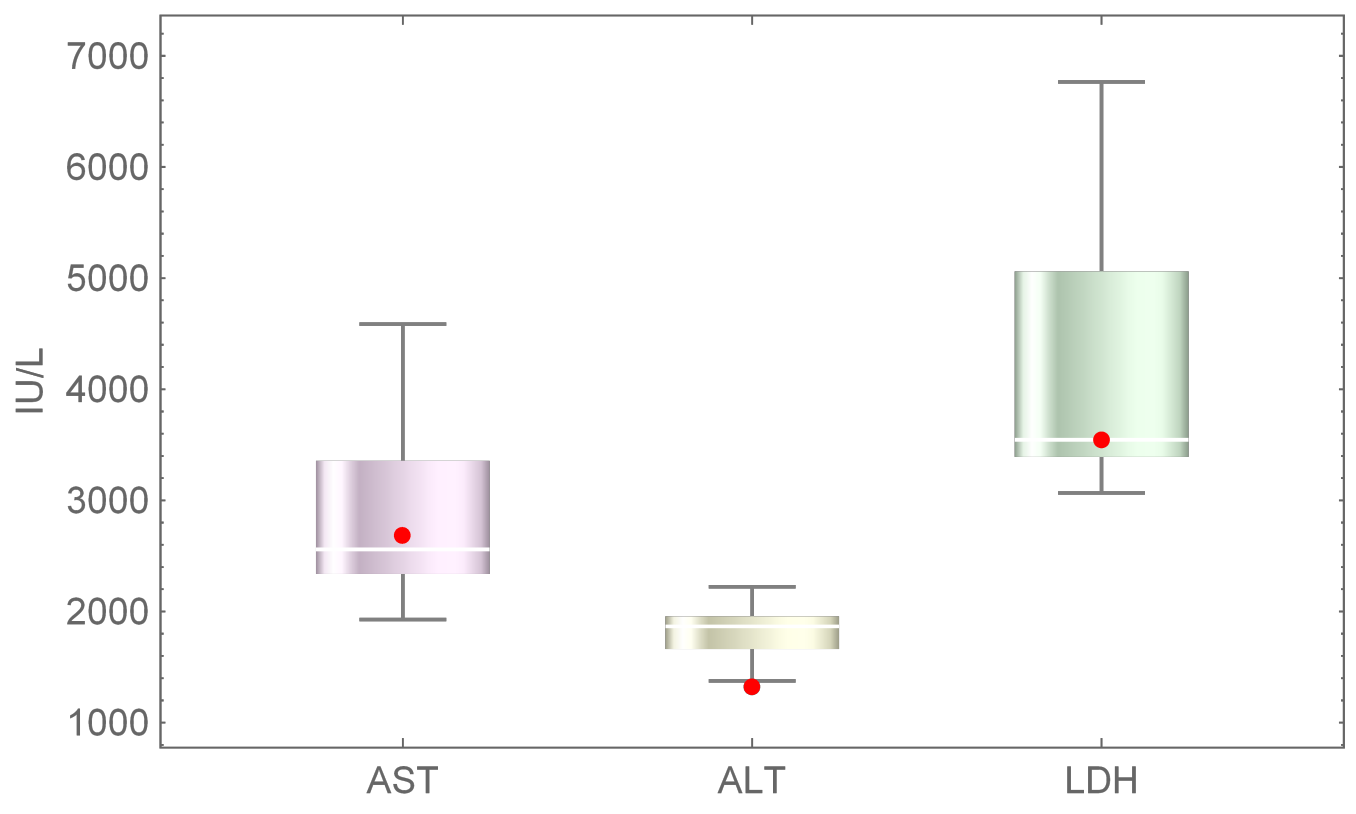

Figure 1 


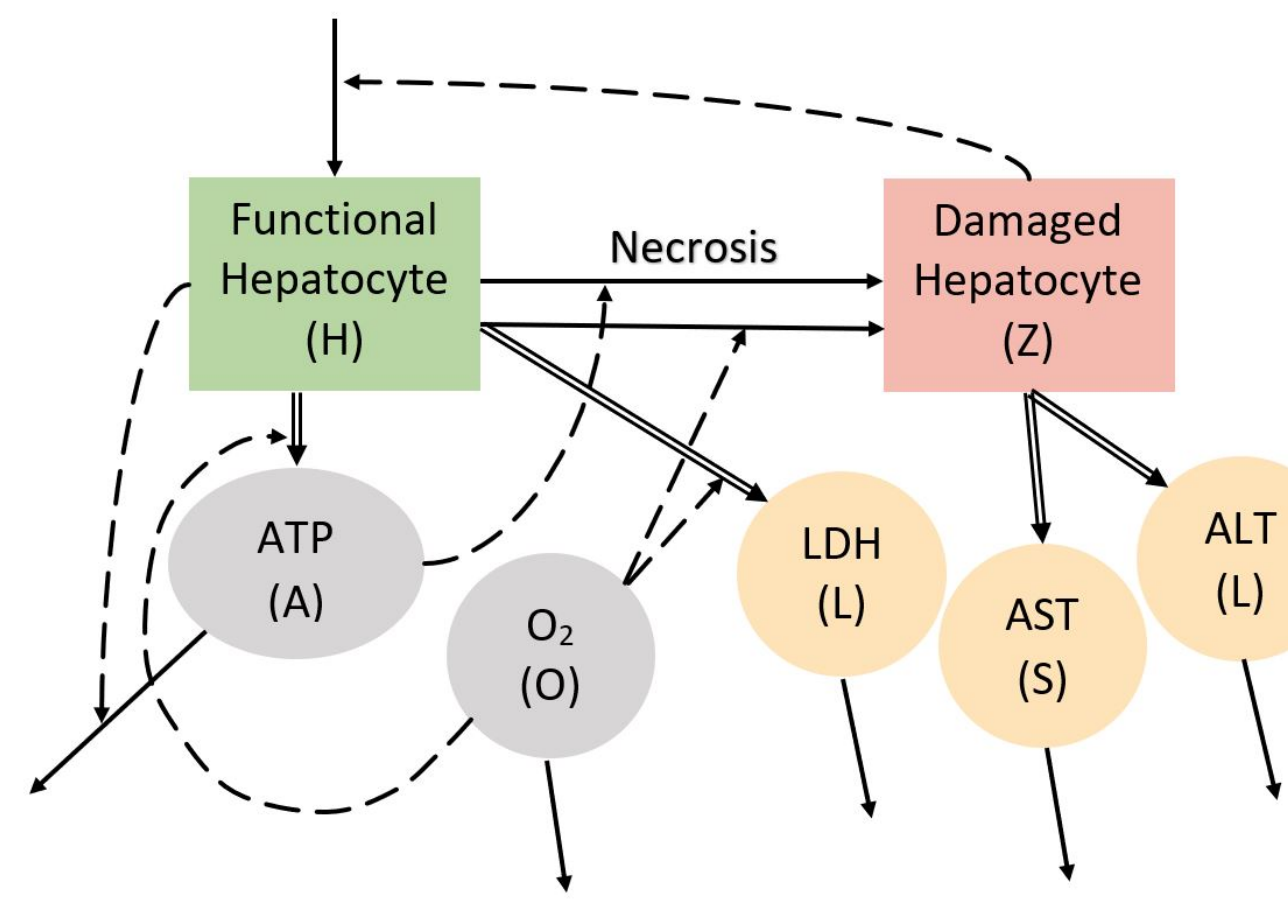

Figure 2: Schematic depiction of the model. Solid arrow indicates conversion of the same variable from one state to another, dotted arrow indicates influence of a variable on the transition rate, and double arrow indicates secretion of biomarkers. See expressions terms of the Model System 1-6 to understand the appropriateness of arrows.

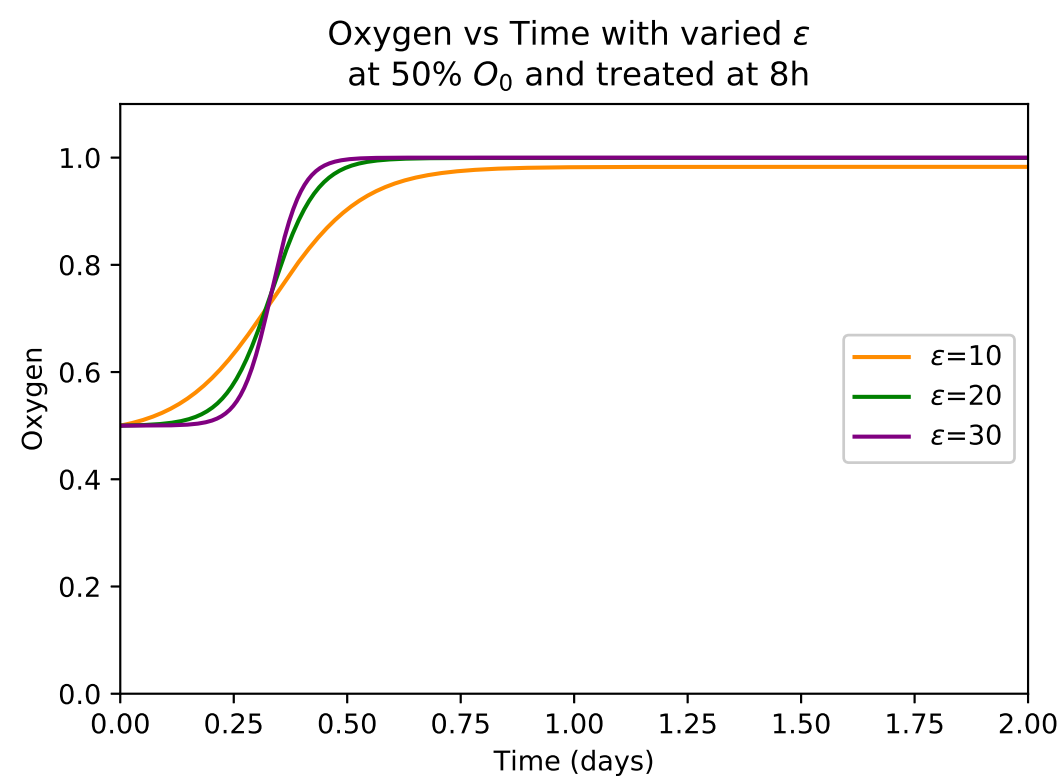

Figure 3: Demonstration of the oxygen function at $50 \%$ initial $O_{0}$ values for different values of $\epsilon$. 


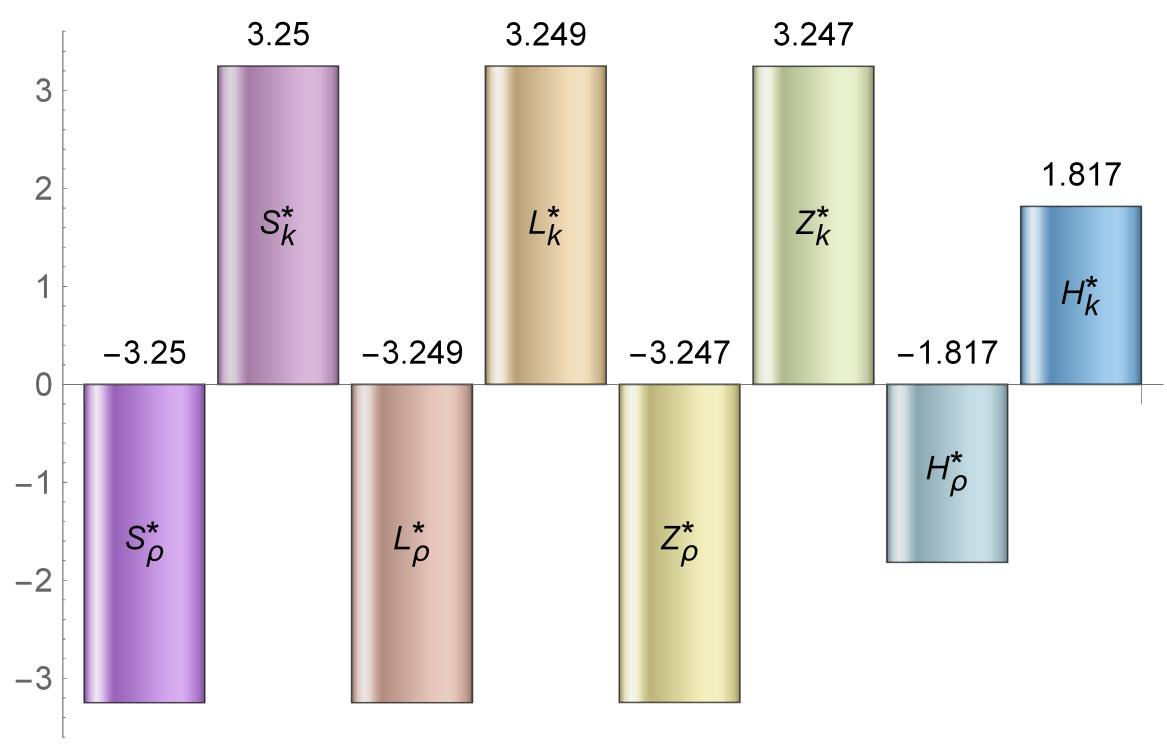

Figure 4: Sensitivity Indexes of the equilibrium concentration of variables with respect to parameters $\rho$ and $k$. Note, $\rho$ negatively and $k$ positively impacts equilibrium concentration of all model variables.

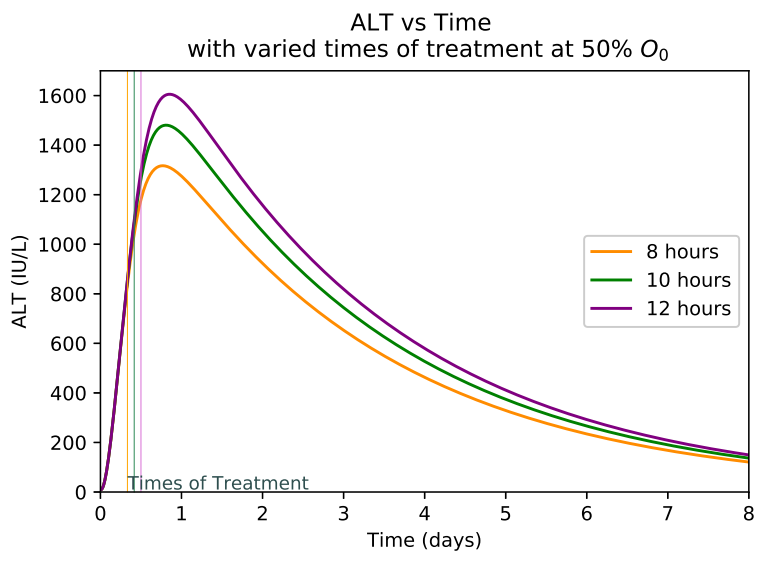

(a) ALT peaks with varied times of treatment at $50 \%$ oxygen level.

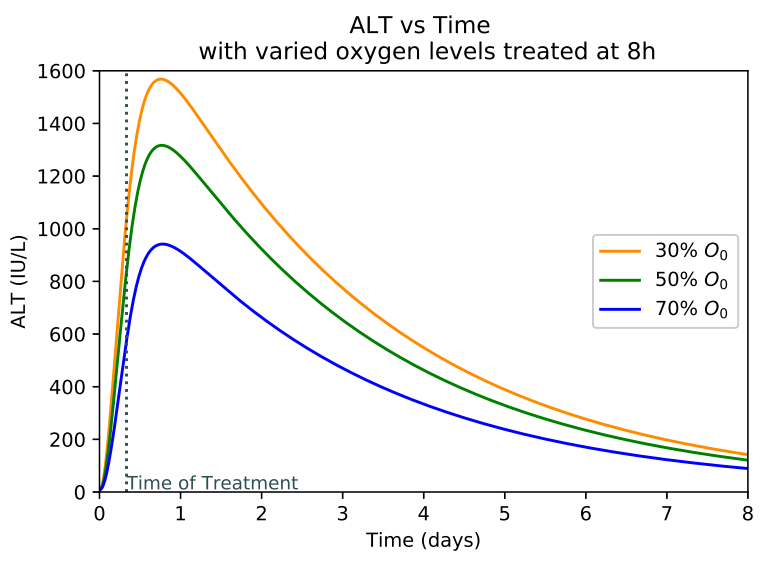

(b) ALT peaks with varied initial oxygen levels treated after 8 hours.

Figure 5: ALT peaks with varied times of treatment and oxygen levels.

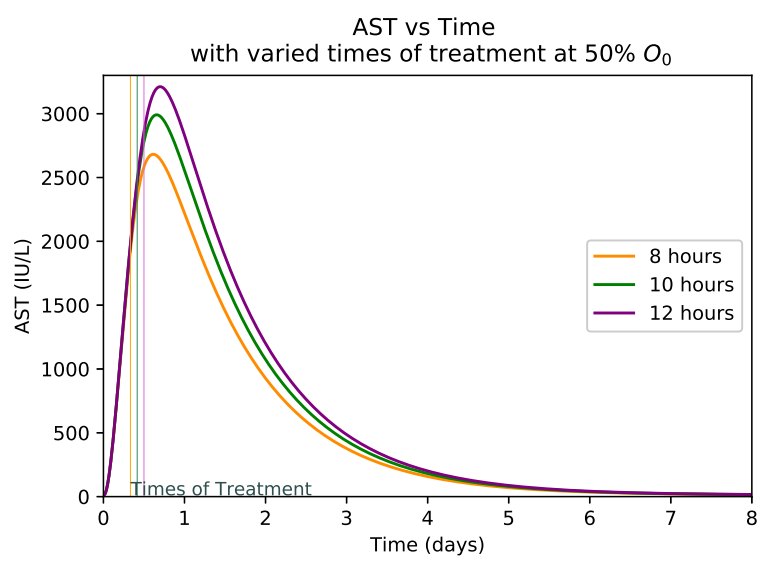

(a) AST peaks with varied times of treatment at $50 \%$ oxygen level.

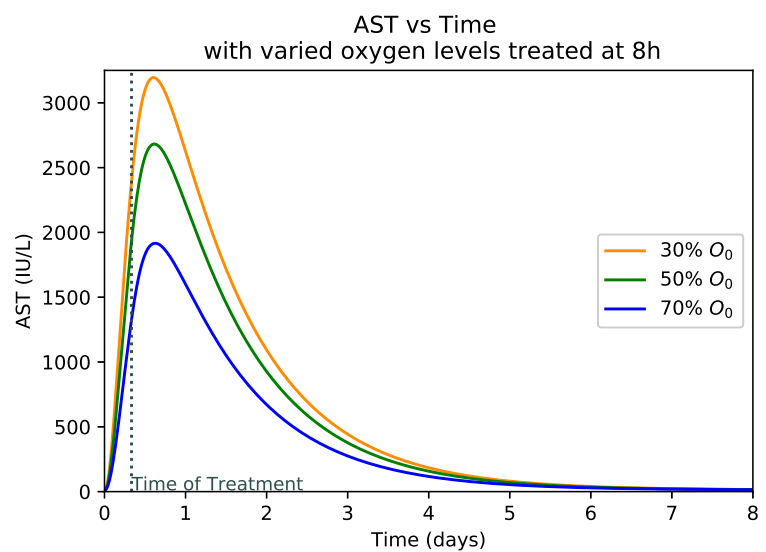

(b) AST peaks with varied initial oxygen levels treated after 8 hours.

Figure 6: AST peaks with varied times of treatment and oxygen levels. 


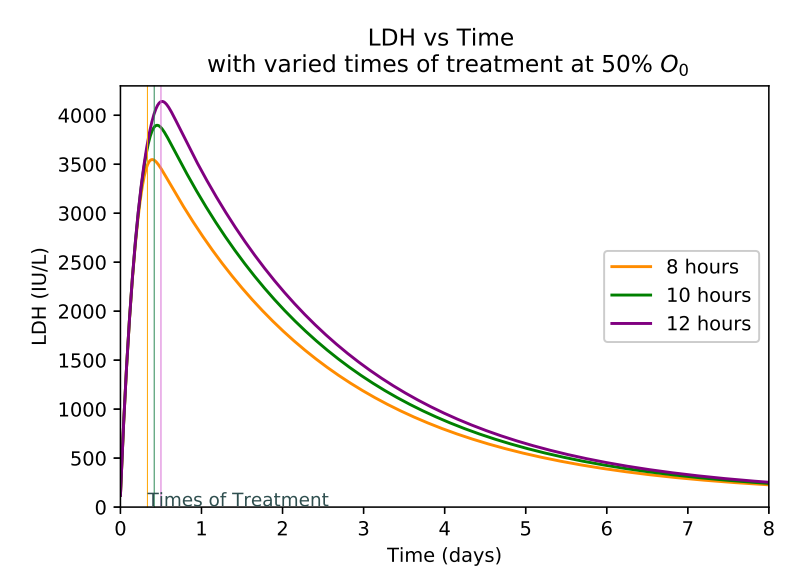

(a) $\mathrm{LDH}$ peaks with varied times of treatment at $50 \%$ oxygen level.

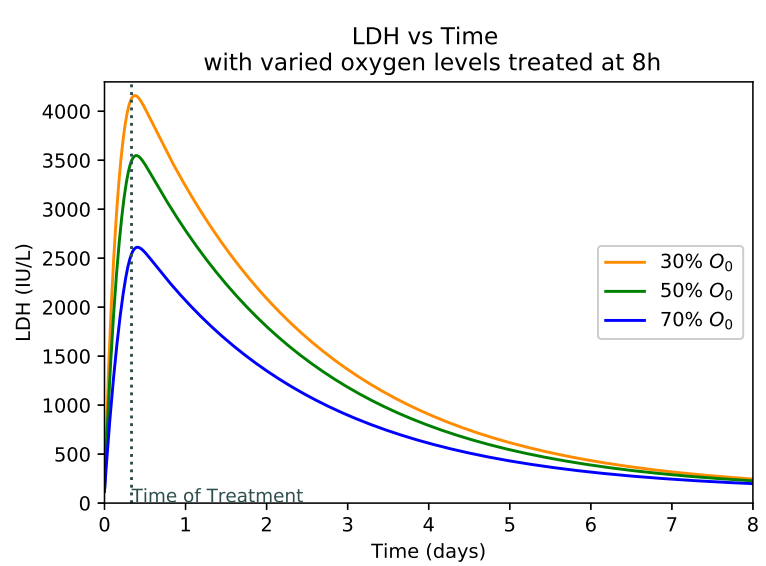

(b) $\mathrm{LDH}$ peaks with varied initial oxygen levels treated after 8 hours.

Figure 7: LDH peaks with varied times of treatment and oxygen levels.

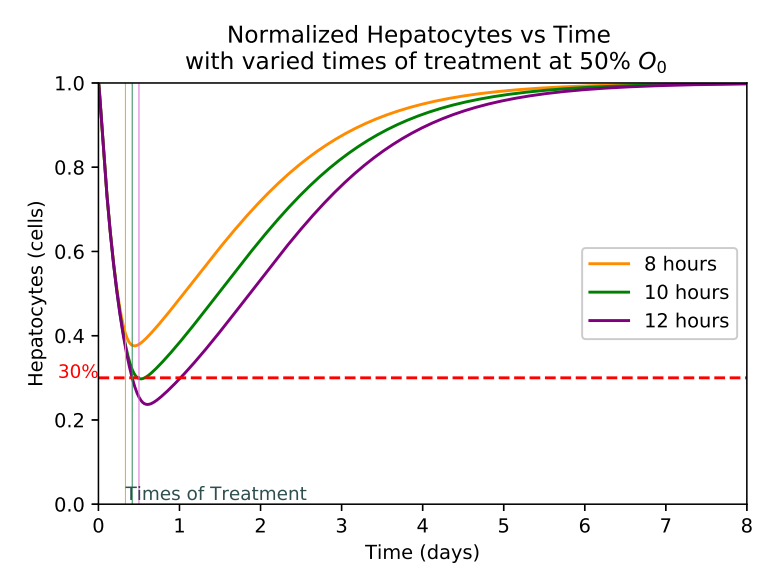

(a) Healthy Hepatocytes as function of normal with var- (b) ied times of treatment at $50 \%$ oxygen level.

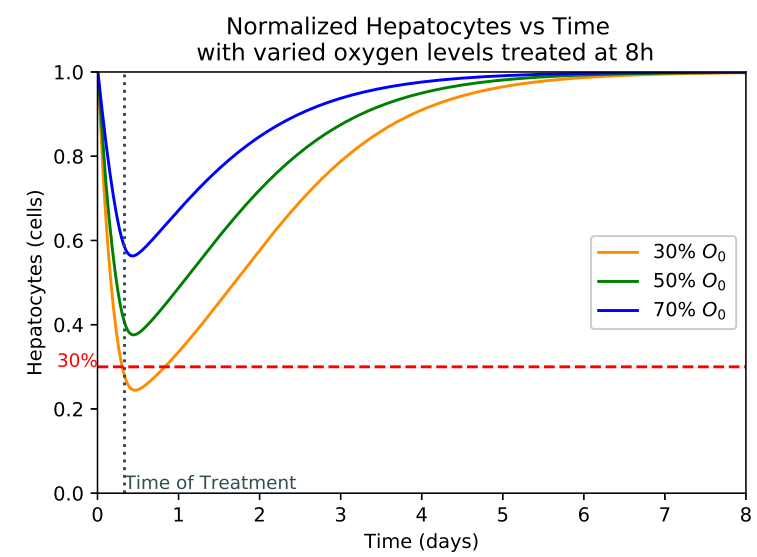

(b) Healthy Hepatocytes as a function of normal with varied initial oxygen levels treated after 8 hours.

Figure 8: Hepatocyte damage with varied times of treatment and oxygen levels.

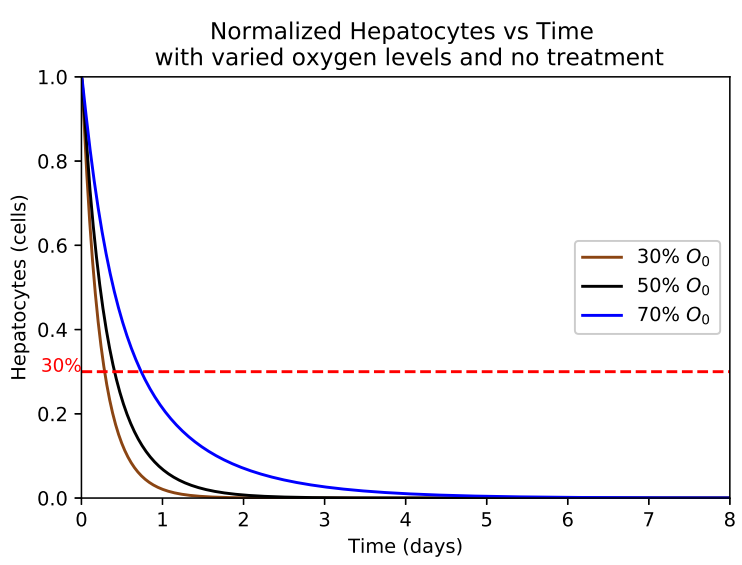

(a) Hepatocyte death with varied O2 levels when there is no treatment.

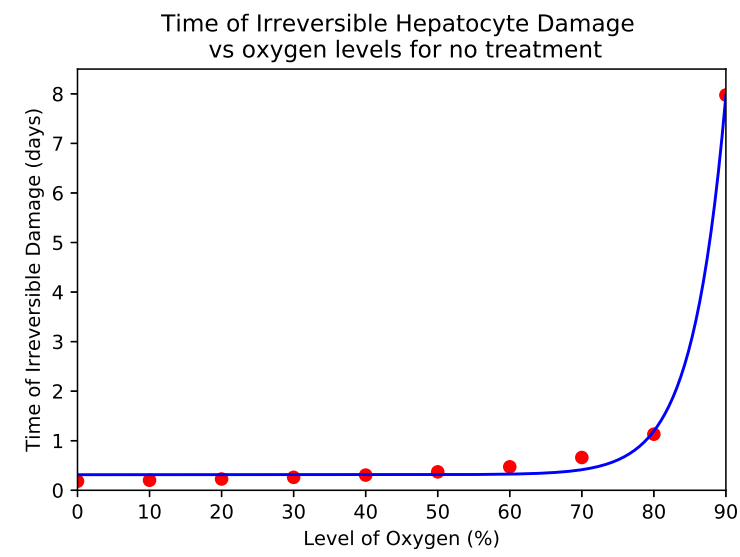

(b) The estimated time of reaching the critical 30\% Hepatocyte level for irreversible damage given the initial oxygen level.

Figure 9: Demonstration of the Hepatocyte, AST, ALT, LDH level with varied O2 levels when there is no treatment. 
bioRxiv preprint doi: https://doi.org/10.1101/2020.03.11.987594; this version posted March 11, 2020. The copyright holder for this preprint (which was not certified by peer review) is the author/funder, who has granted bioRxiv a license to display the preprint in perpetuity. It is made available under aCC-BY 4.0 International license.

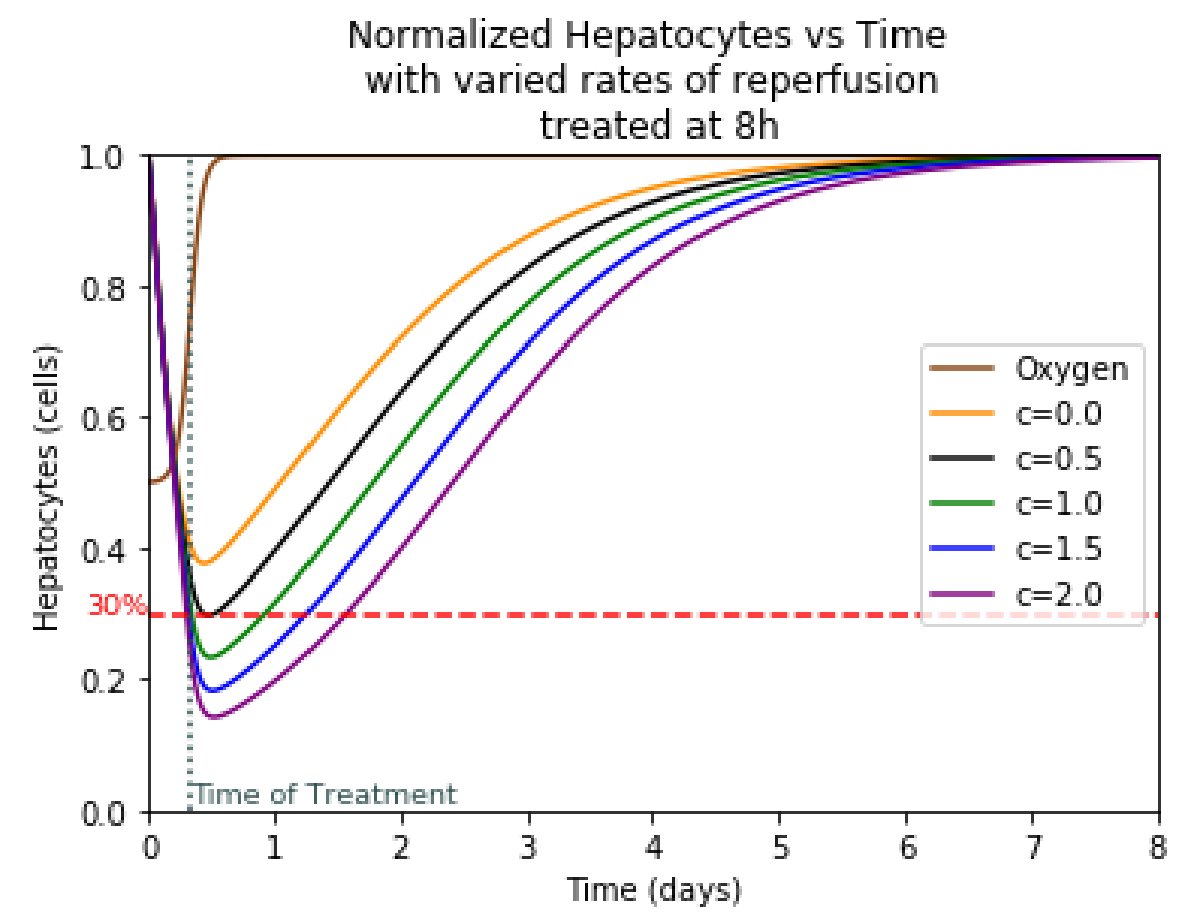

Figure 10: Hepatocyte damage with varied rates of reperfusion treated at the 8th hour. 
Table 1: Table of Variables

\begin{tabular}{|c|c|c|c|}
\hline Variables & Value & Description & \\
\hline 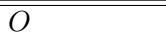 & unitless & Amount of oxygen & \\
\hline$A$ & pmol & Amount of total ATP in the liver & \\
\hline$H$ & cells & Number of healthy hepatocytes in a liver & \\
\hline$Z$ & cells & Number of damaged hepatocytes in a liver & \\
\hline$S$ & $I U / L$ & Amount of AST per liter of blood & \\
\hline$L$ & $I U / L$ & Amount of ALT per liter of blood & \\
\hline$D$ & $I U / L$ & Amount of LDH per liter of blood & \\
\hline Parameters & Value & Description & Source \\
\hline$\overline{A_{\text {norm }}}$ & $1.6 \times 10^{9} \mathrm{pmol}$ & Amount of ATP in a healthy liver & 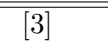 \\
\hline$\rho$ & $1.43 \mathrm{pmol} / \mathrm{cell} / \mathrm{day}$ & ATP production rate & {$[15]$} \\
\hline$k$ & $1.43 \mathrm{pmol} / \mathrm{cell} / \mathrm{day}$ & ATP consumption rate & {$[15]$} \\
\hline$r$ & 1 days $^{-1}$ & Functional hepatocyte regeneration rate & {$[36]$} \\
\hline$\eta$ & 6.381 days $^{-1}$ & Cell necrosis rate & {$[2]$} \\
\hline$\delta_{Z}$ & 5 days $^{-1}$ & Damaged hepatocyte lysis rate & {$[36]$} \\
\hline$H_{\max }$ & $1.6 \times 10^{11} \mathrm{cells}$ & $\begin{array}{l}\text { Maximum number of hepatocytes possible in a healthy liver } \\
\text { (assumed constant) }\end{array}$ & {$[36]$} \\
\hline$\theta$ & $5 L$ & Total amount of blood in human body & {$[36]$} \\
\hline$\delta_{S}$ & 0.92 days $^{-1}$ & AST clearance rate & {$[36]$} \\
\hline$\delta_{L}$ & 0.35 days $^{-1}$ & ALT clearance rate & {$[36]$} \\
\hline$\delta_{D}$ & 0.459 days $^{-1}$ & LDH clearance rate & {$[5]$} \\
\hline$\beta_{S}$ & $20000 I U$ & Total amount of AST in a healthy liver & {$[20,34]$} \\
\hline$\beta_{L}$ & $9000 I U$ & Total amount of ALT in a healthy liver & {$[20,34]$} \\
\hline$\beta_{D}$ & $200000 I U$ & $\mathrm{LDH}$ production rate & {$[18]$} \\
\hline$S_{\min }$ & $12 I U / L$ & Minimum AST level & {$[36]$} \\
\hline$L_{\min }$ & $9 \mathrm{IU} / \mathrm{L}$ & Minimum ALT level & {$[36]$} \\
\hline$D_{\min }$ & $120 I U / L$ & Minimum LDH level & {$[16]$} \\
\hline$D_{\max }$ & $30000 \mathrm{IU} / L$ & Maximum LDH level & {$[20]$} \\
\hline$O_{0}$ & varied & Oxygen fraction from cardiac injury & Estm. \\
\hline$\epsilon$ & 20 days $^{-1}$ & Rate of oxygen return & {$[33]$} \\
\hline$\tau$ & days & Time of treatment & Estm. \\
\hline
\end{tabular}


Time of Irreversible Hepatocyte Damage vs oxygen levels for no treatment

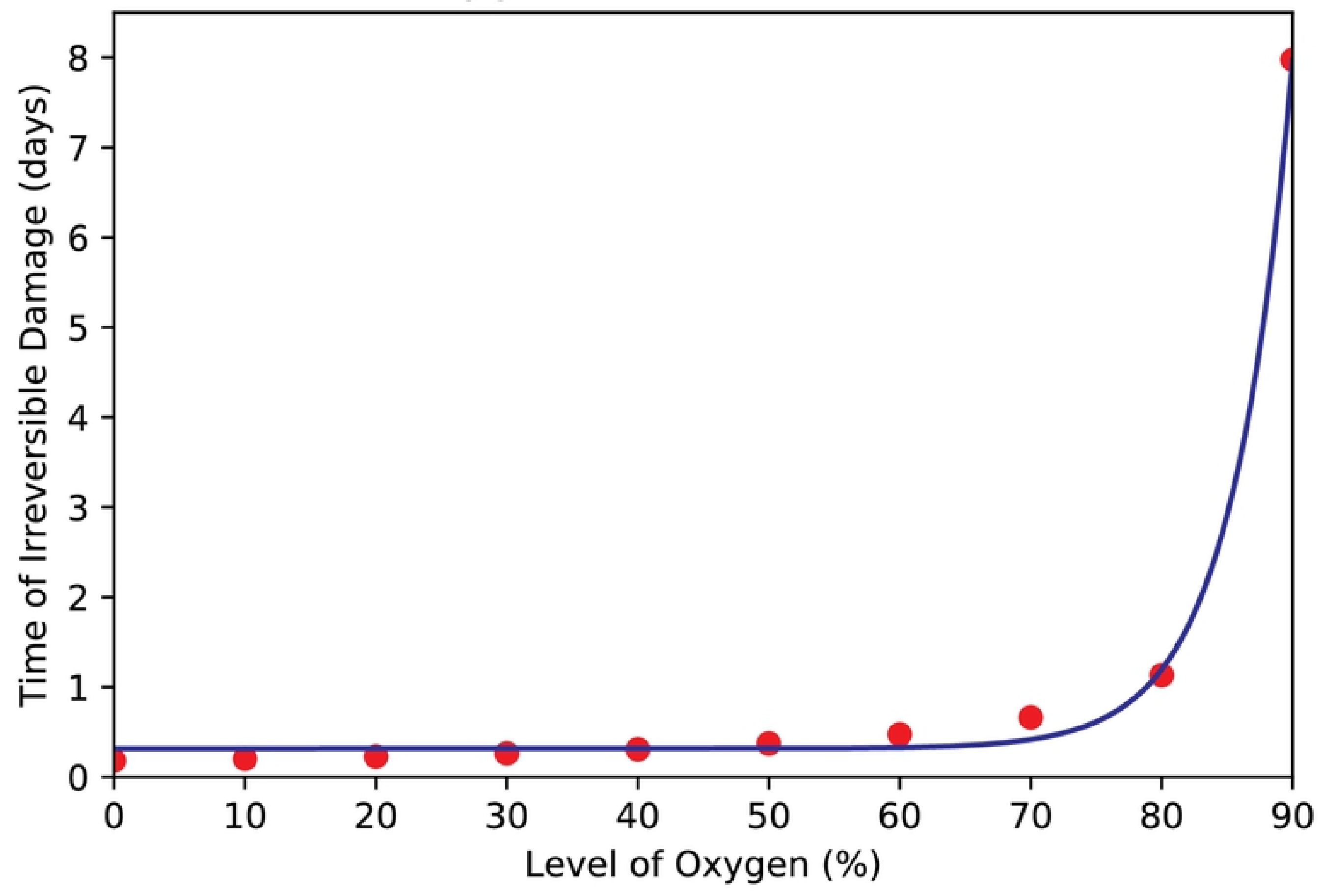

Figure 
ALT vs Time

with varied times of treatment at $50 \% \mathrm{O}_{0}$

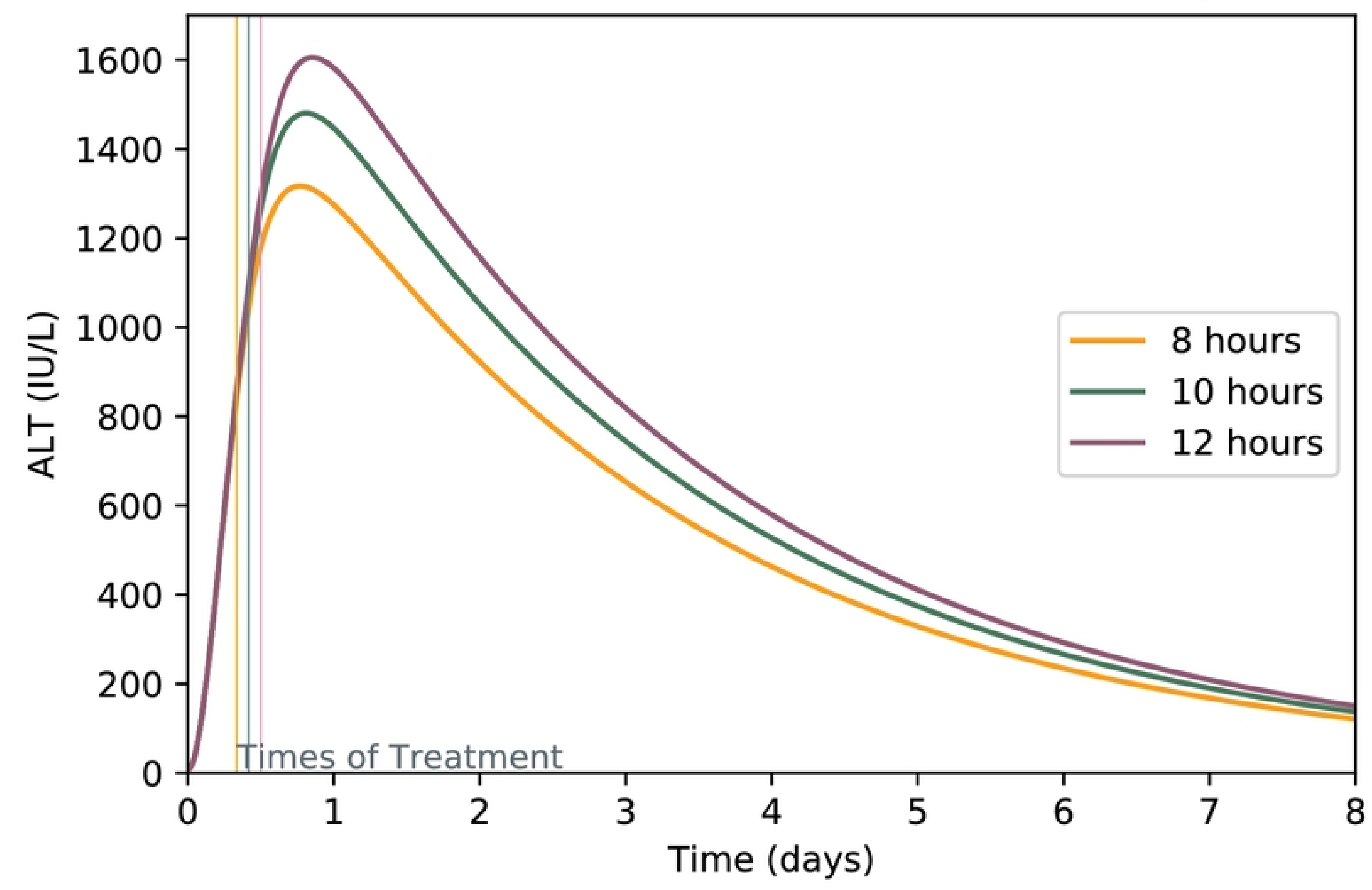

Figure 
ALT vs Time

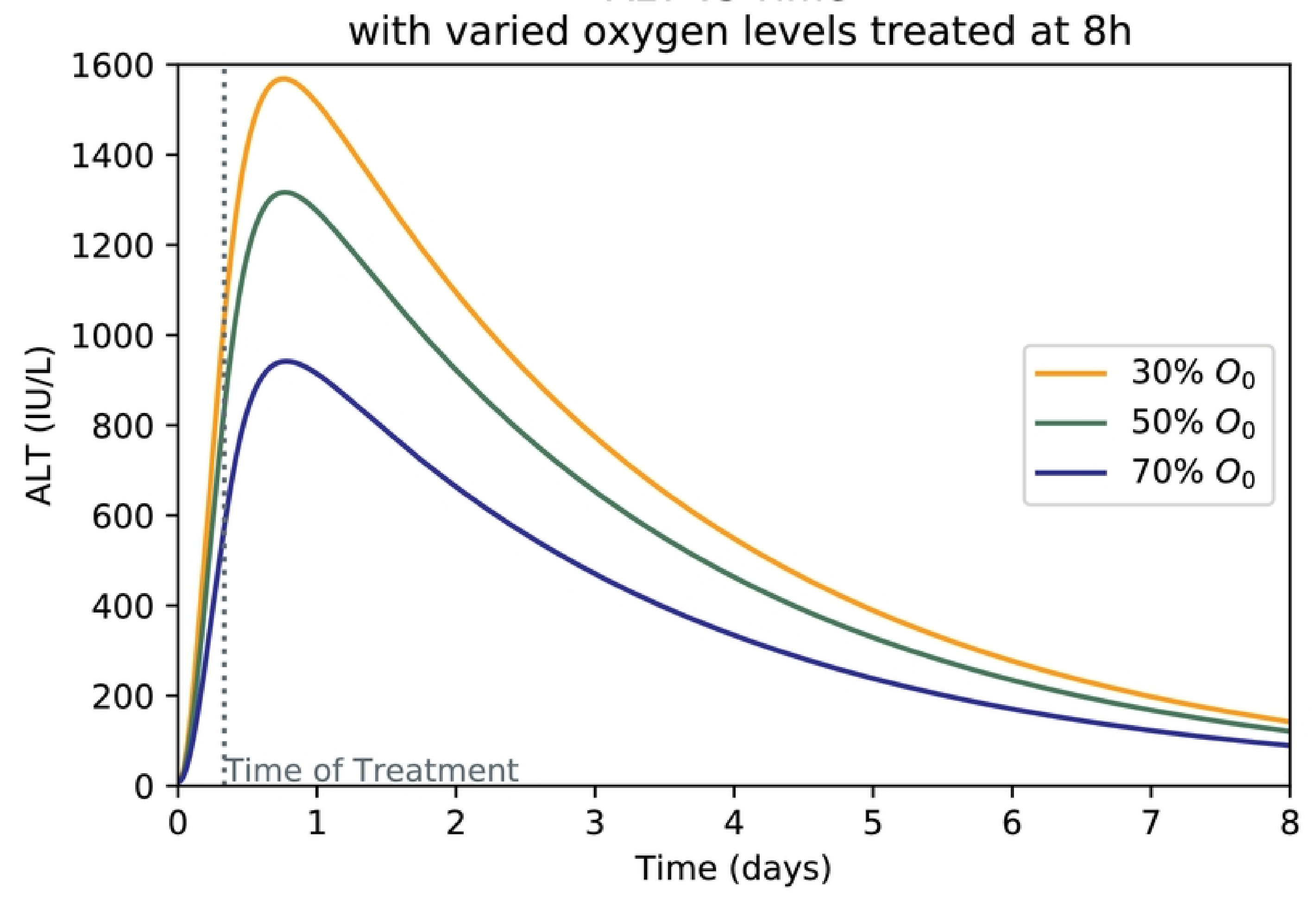

Figure 


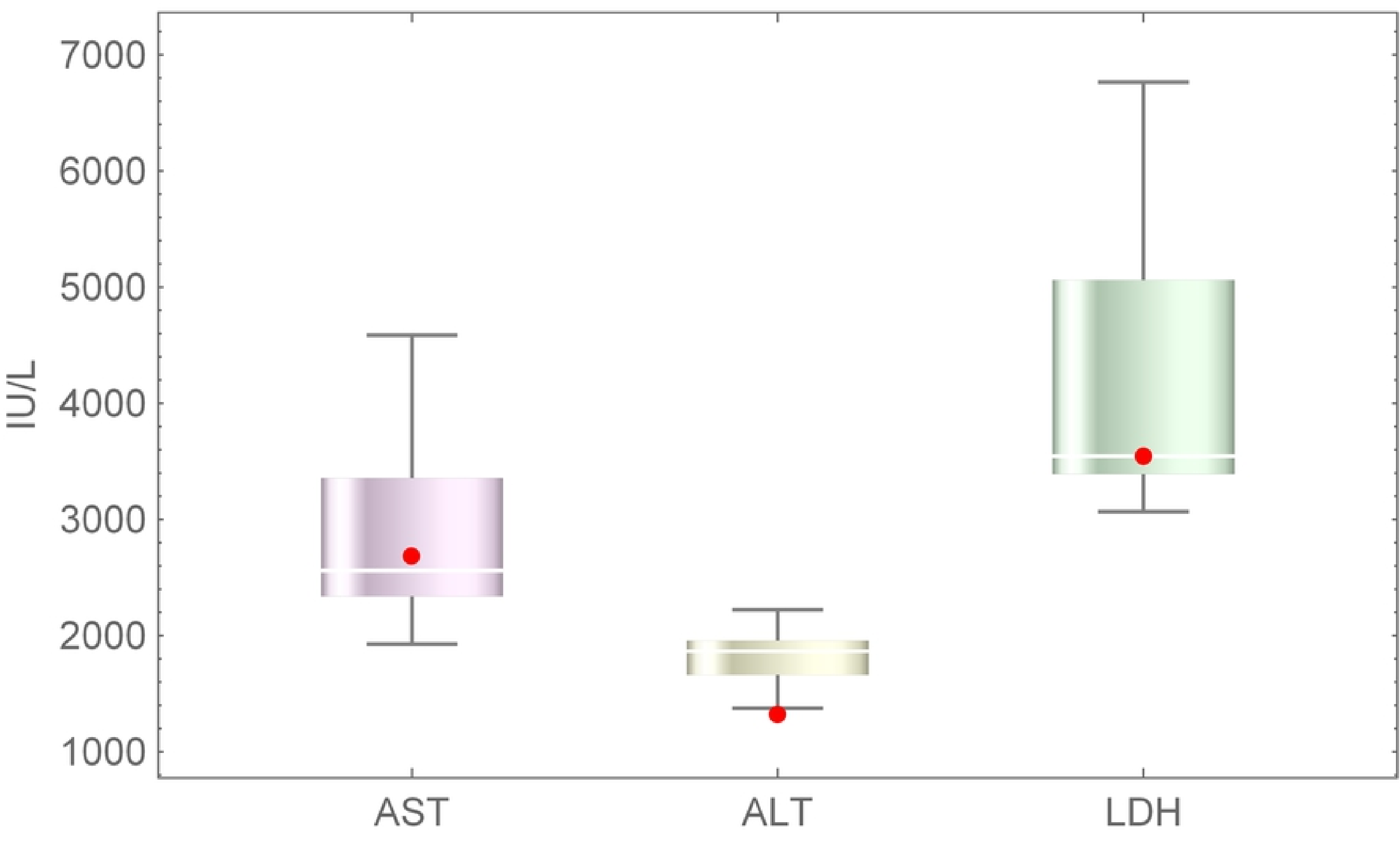

Figure 
with varied times of treatment at $50 \% O_{0}$

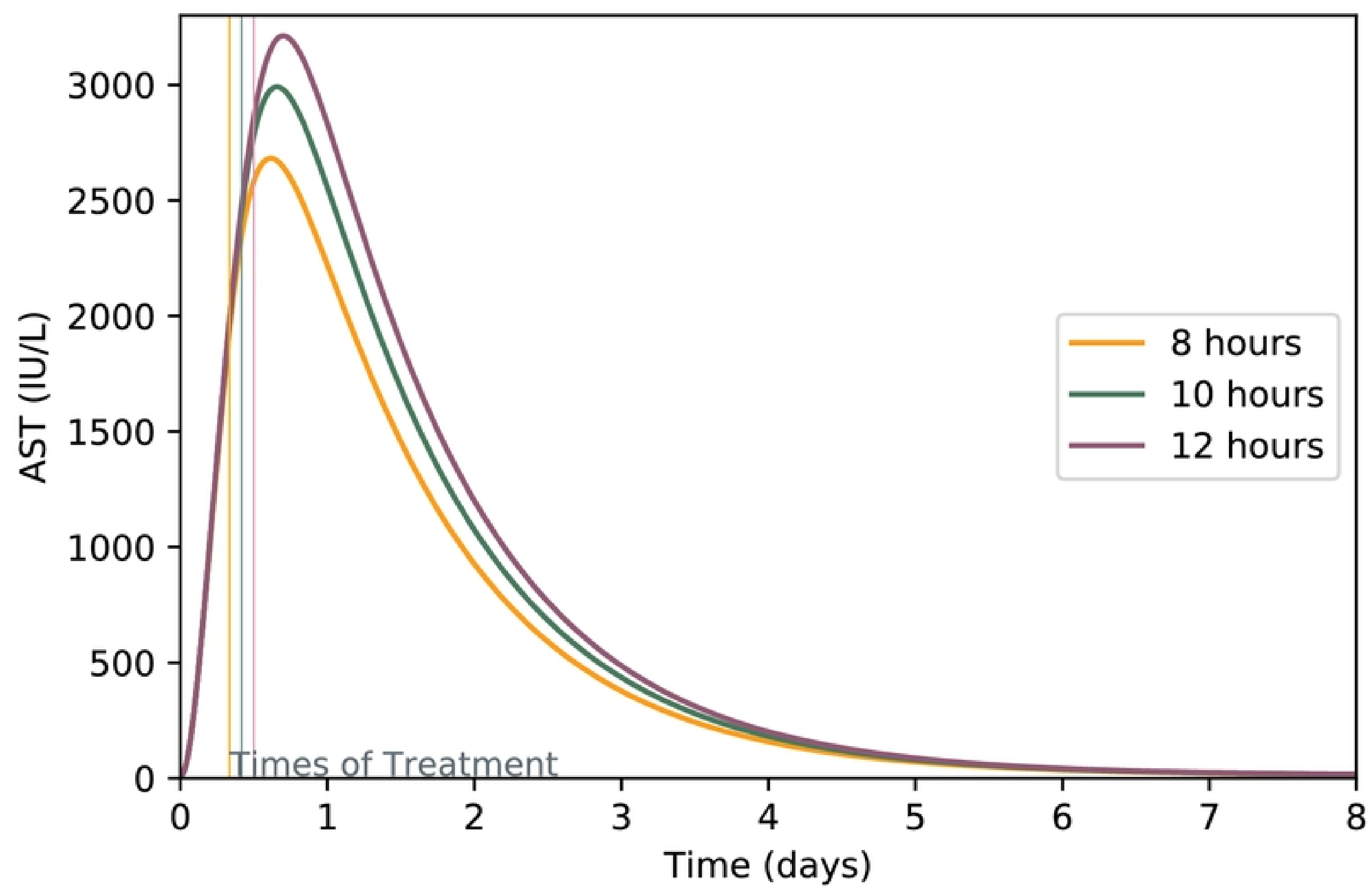

Figure 
AST vs Time

with varied oxygen levels treated at $8 \mathrm{~h}$

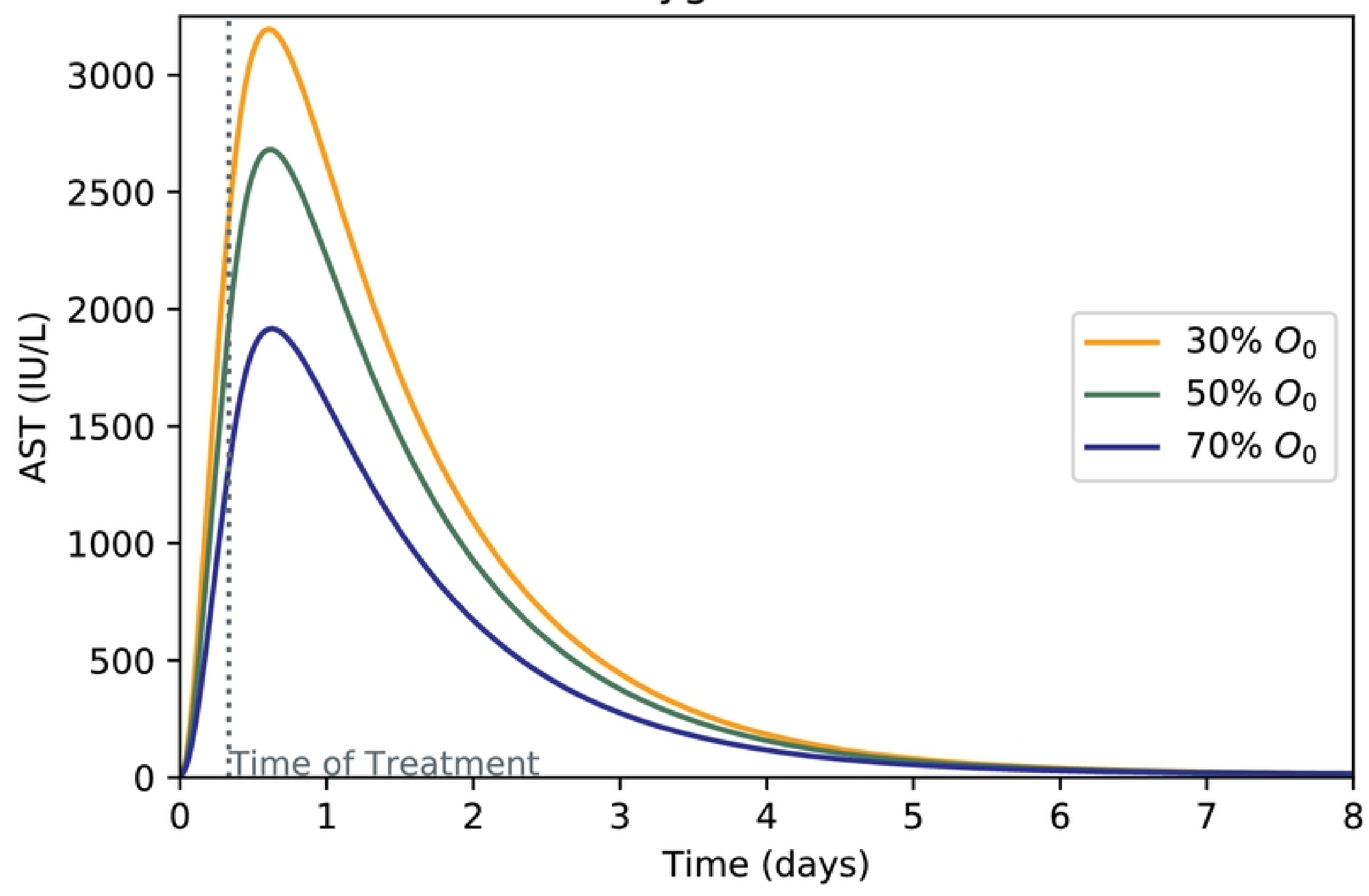

Figure 


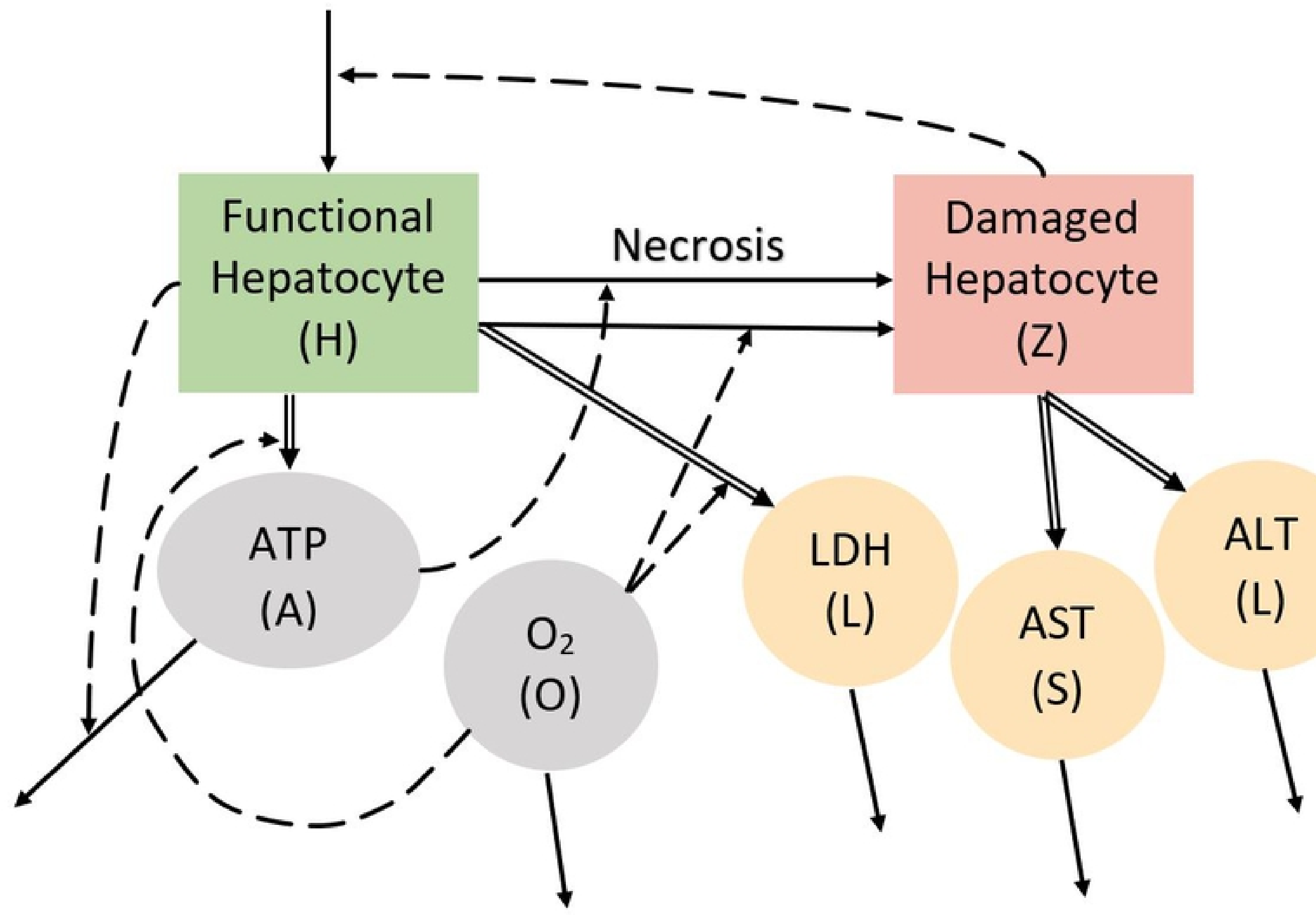

Figure 
with varied times of treatment at $50 \% \mathrm{O}_{0}$

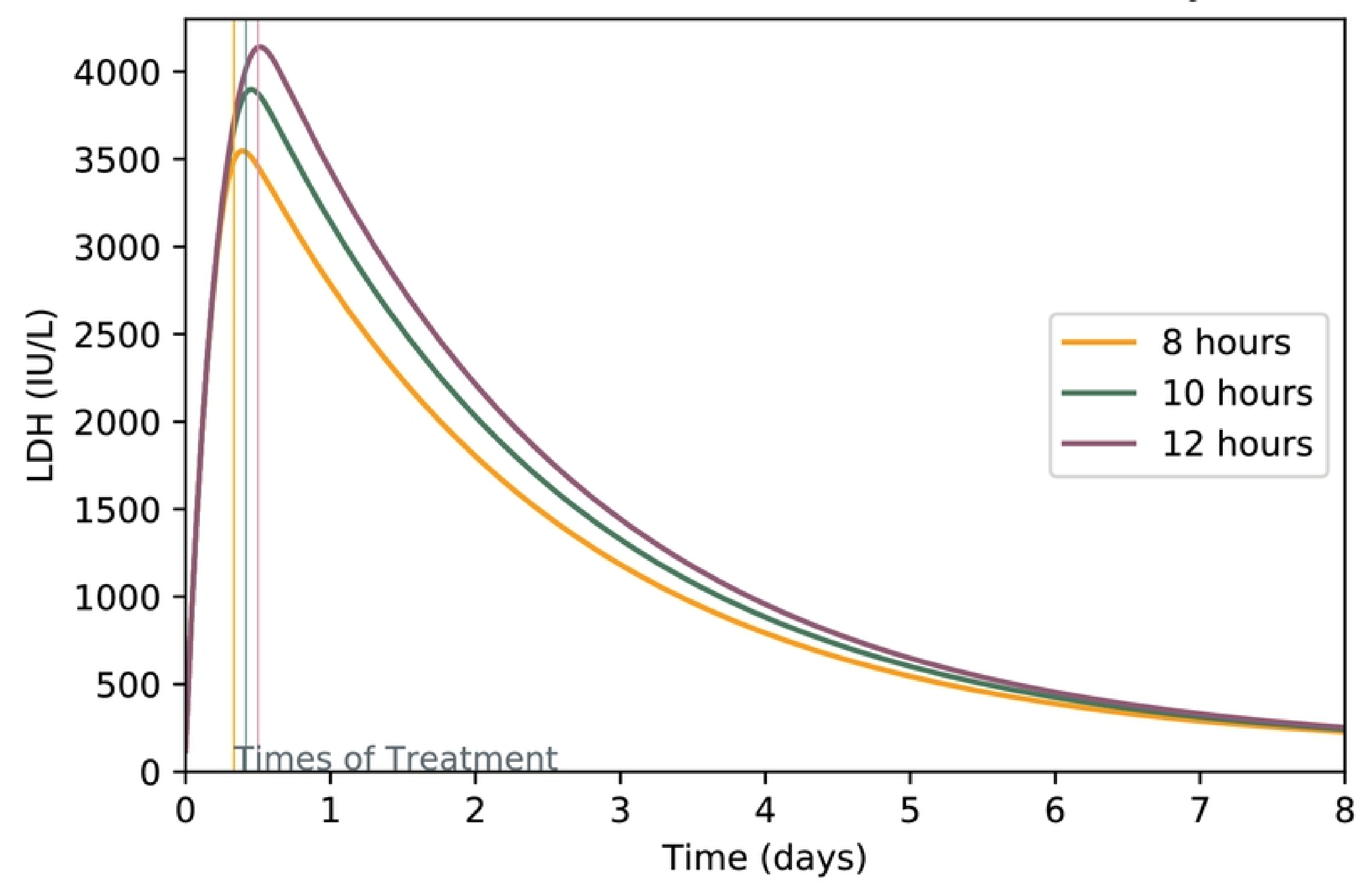

Figure 


\section{LDH vs Time}

with varied oxygen levels treated at $8 \mathrm{~h}$

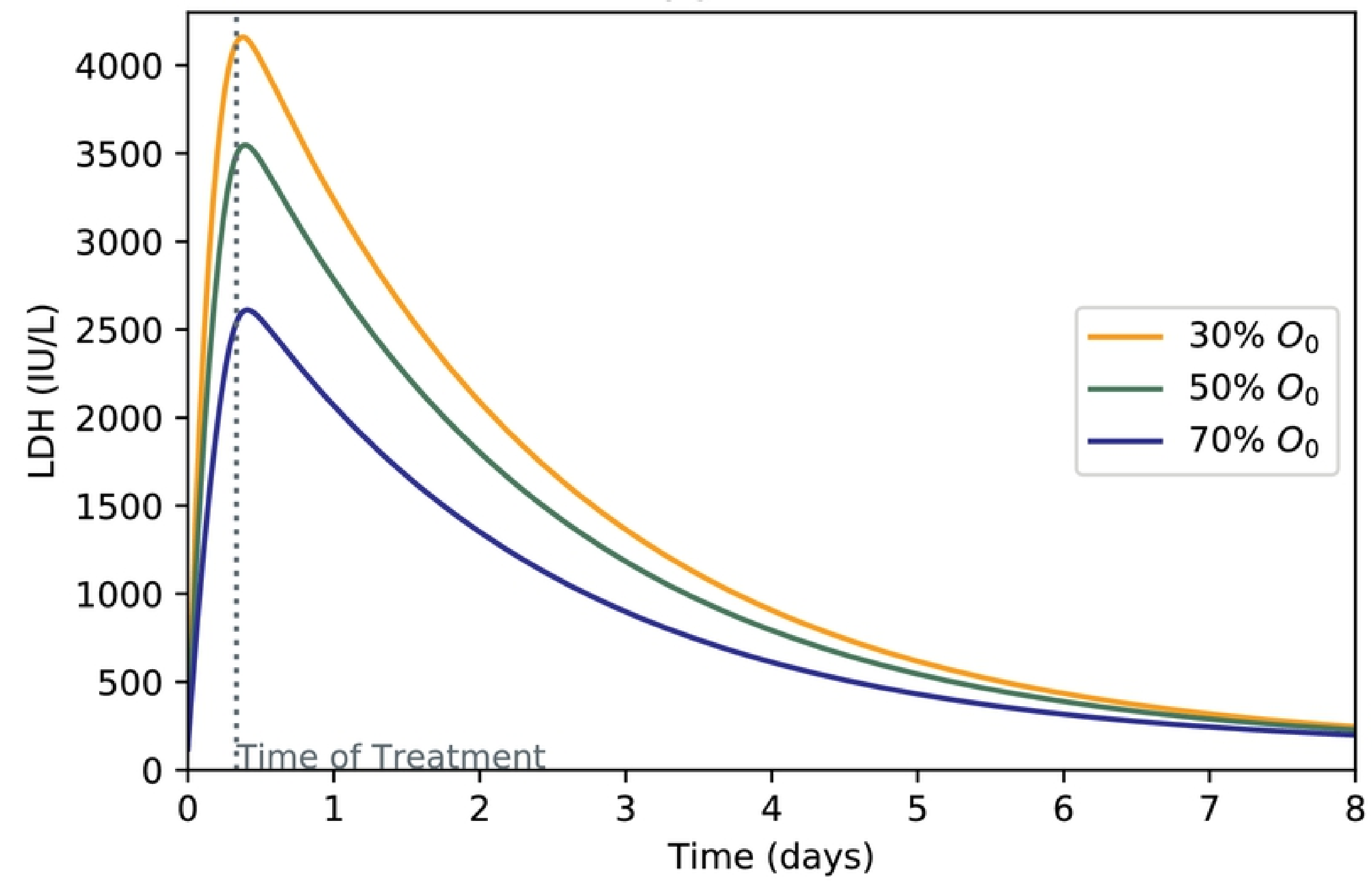

Figure 
Normalized Hepatocytes vs Time

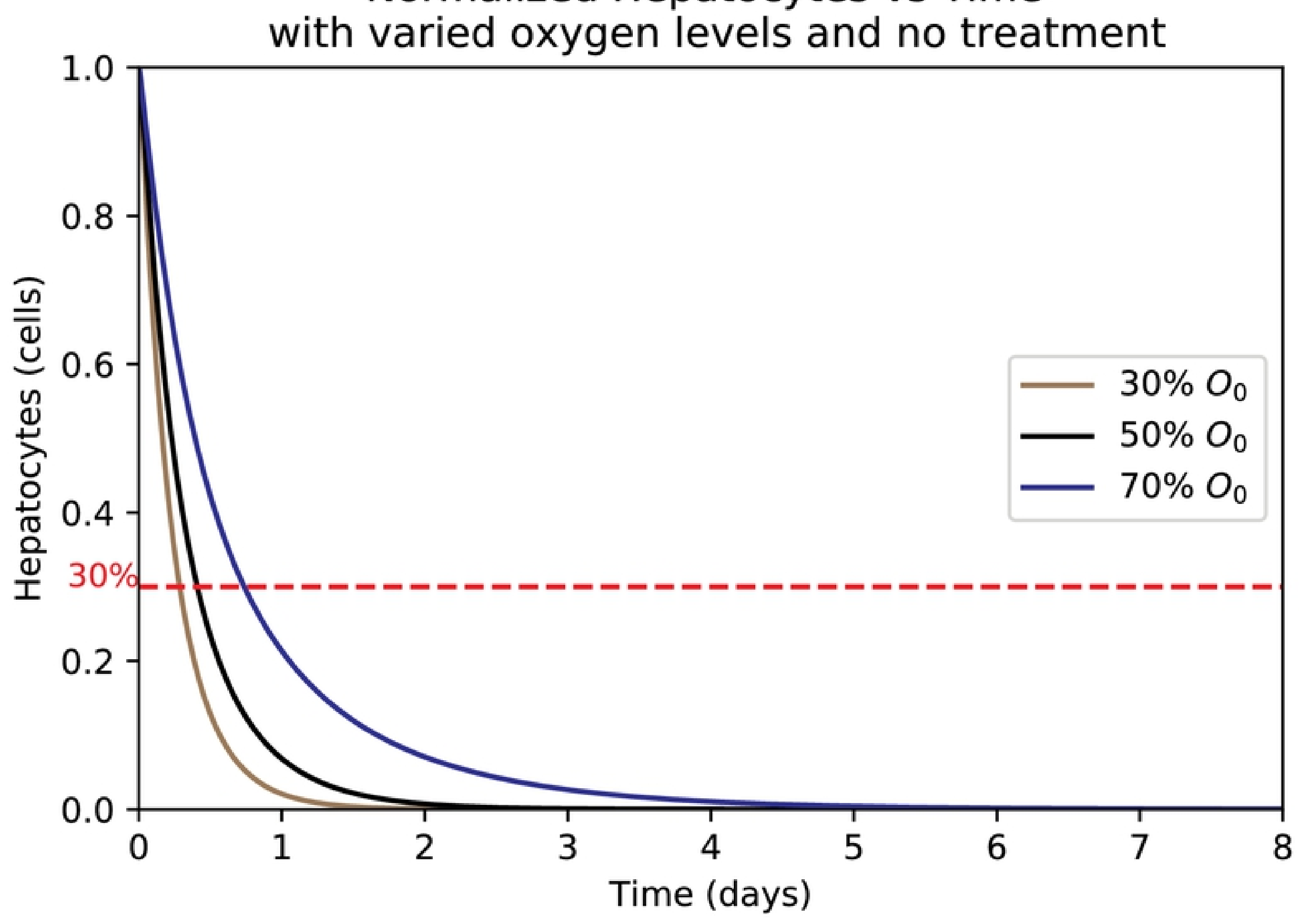

Figure 
Oxygen vs Time with varied $\varepsilon$ at $50 \% O_{0}$ and treated at $8 \mathrm{~h}$

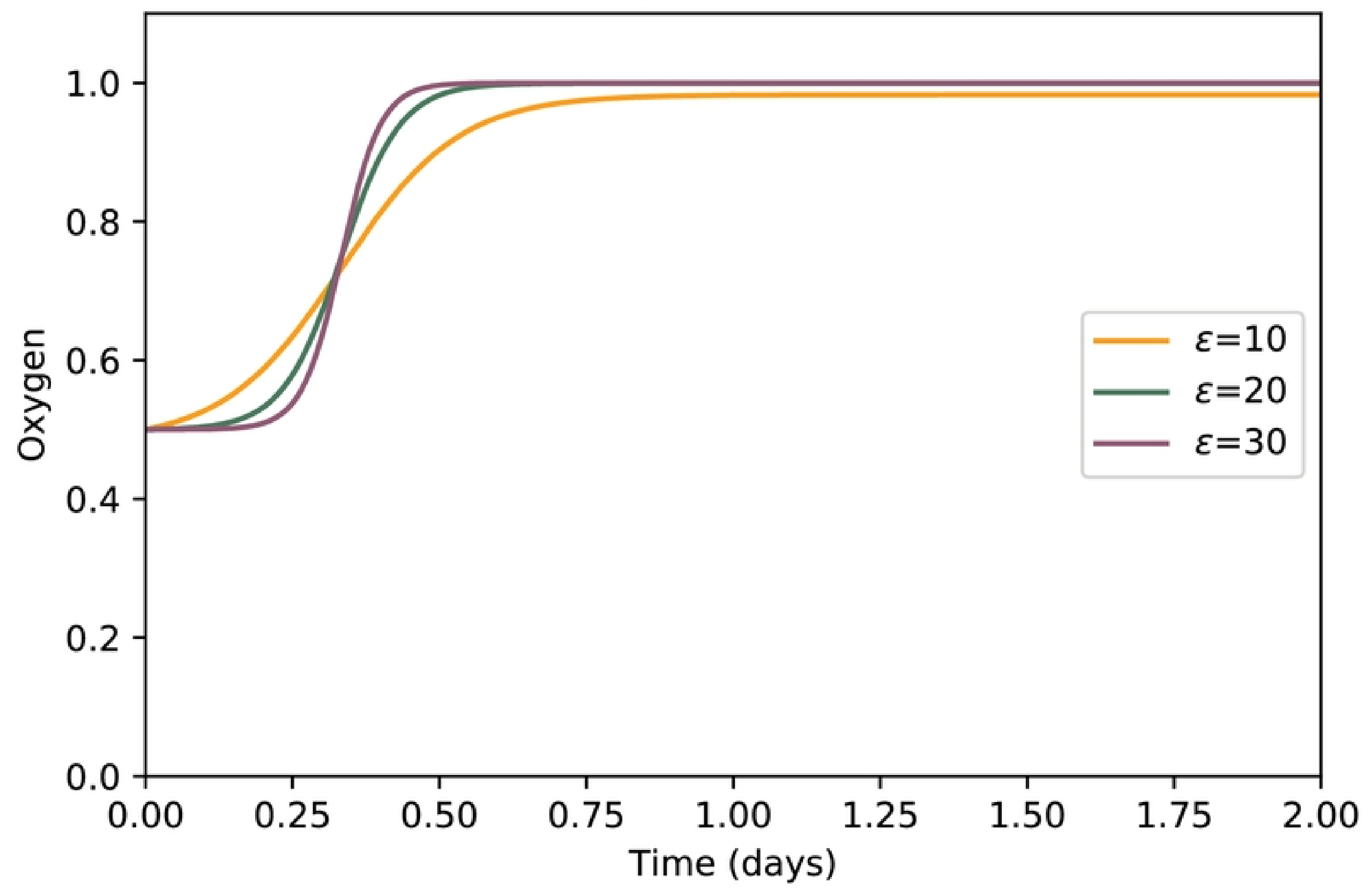

Figure 


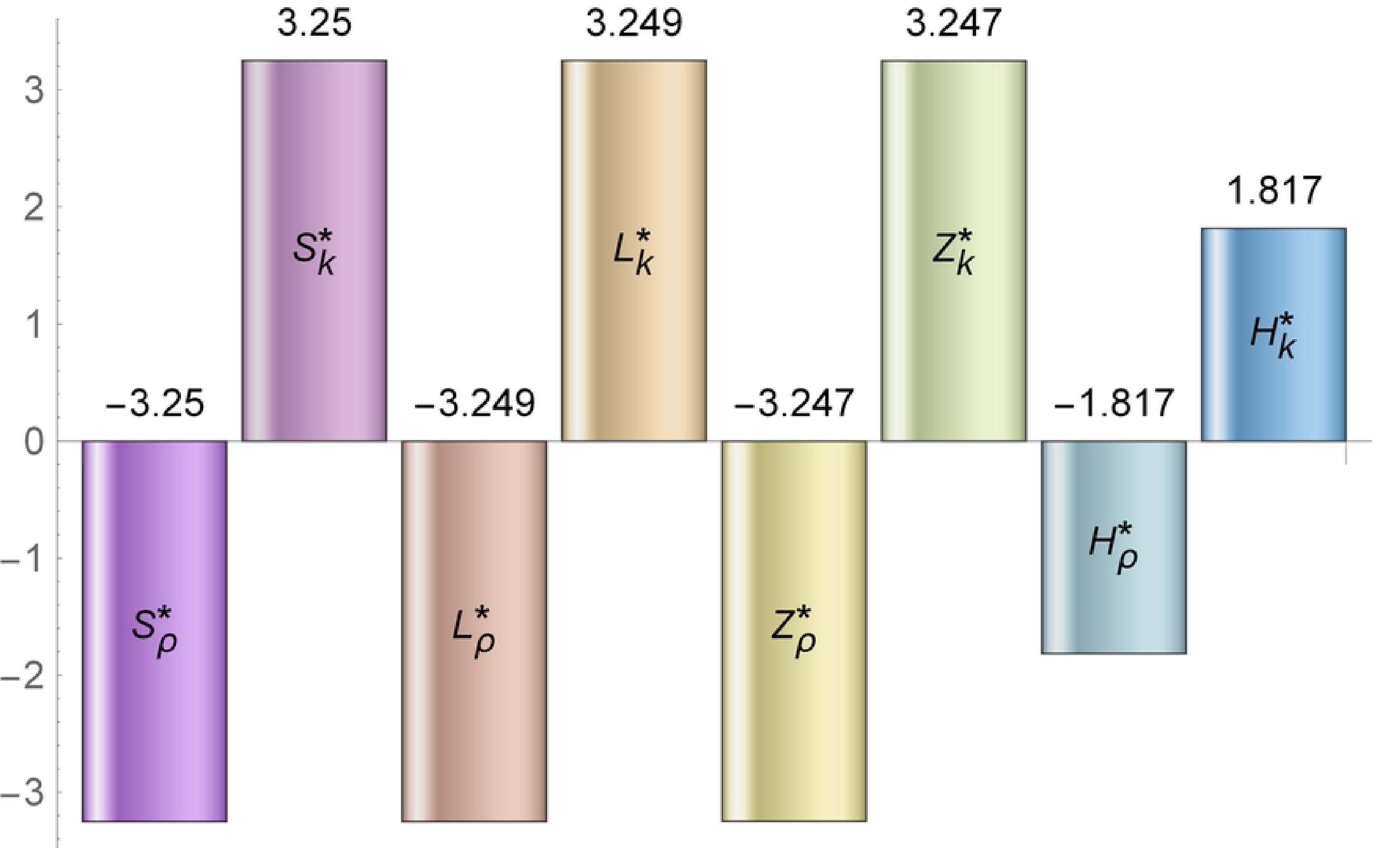

Figure 